\title{
RATIONALITY OF THE GENERALIZED BINOMIAL COEFFICIENTS FOR A MULTIPLICITY FREE ACTION
}

\author{
CHAL BENSON and GAIL RATCLIFF
}

(Received 14 October 1998; revised 19 November 1999)

\author{
Communicated by T. Dooley
}

\begin{abstract}
Let $V$ be a finite dimensional Hermitian vector space and $K$ be a compact Lie subgroup of $U(V)$ for which the representation of $K$ on $\mathbb{C}[V]$ is multiplicity free. One obtains a canonical basis $\left\{p_{\alpha}\right\}$ for the space $\mathbb{C}\left[V_{R}\right]^{K}$ of $K$-invariant polynomials on $V_{\mathbb{R}}$ and also a basis $\left\{q_{\alpha}\right\}$ via orthogonalization of the $p_{\alpha}$ 's. The polynomial $p_{\alpha}$ yields the homogeneous component of highest degree in $q_{\alpha}$. The coefficients that express the $q_{\alpha}$ 's in terms of the $p_{\beta}$ 's are the generalized binomial coefficients of Yan. The main result in this paper shows that these numbers are rational.
\end{abstract}

2000 Mathematics subject classification: primary 05E35, $20 \mathrm{G} 05$.

\section{Introduction}

Throughout this paper, $V$ denotes a finite dimensional complex vector space with Hermitian inner product $\langle\cdot, \cdot\rangle$ and norm $|\cdot|$. $K$ denotes a compact Lie group which acts linearly and unitarily on $V$. We write $k z$ for the action of $k \in K$ on a vector $z \in V$. The associated representation of $K$ on the space $\mathbb{C}[V]$ of holomorphic polynomials on $V$ is given by the formula $(k \cdot p)(z)=p\left(k^{-1} z\right)$. The action of $K$ on $V$ is said to be a (linear) multiplicity free action when this representation of $K$ on $\mathbb{C}[V]$ is multiplicity free. That is, no irreducible representation of $K$ occurs more than once in $\mathbb{C}[V]$. We assume the action of $K$ on $V$ is multiplicity free throughout this paper. As shown in [1, Proposition 2.2], it follows that the action of the identity component of $K$ on $V$ is also multiplicity free. Thus we can assume that $K$ is connected. The multiplicity

This work was supported in part by the University of Missouri Research Board.

(C) 2000 Australian Mathematical Society 0263-6115/2000\$A2.00+0.00 
free actions have been completely classified (see $[5,12,13])$. We make use of this classification below in Section 4 of this paper.

We write the decomposition of $\mathbb{C}[V]$ into $K$-irreducible subspaces as

$$
\mathbb{C}[V]=\sum_{\alpha \in \Lambda} P_{\alpha}
$$

Here $\Lambda$ denotes a countably infinite index set. In Section 2 , we specify $\Lambda$ concretely as a set of highest weights. Decomposition (1.1) is canonical because the action of $K$ is multiplicity free. The subspace $\mathscr{P}_{m}(V) \subset \mathbb{C}[V]$ of homogeneous polynomials of degree $m$ is $K$-invariant. Thus each irreducible $P_{\alpha}$ is contained in some $\mathscr{P}_{m}(V)$. We write $|\alpha|$ for the degree of homogeneity of $P_{\alpha}$, so that $P_{\alpha} \subset \mathscr{P}_{|\alpha|}(V)$.

There are no non-constant $K$-invariant polynomials in $\mathbb{C}[V]$. Indeed, the trivial representation of $K$ occurs only once in $\mathbb{C}[V]$. There are, however, non-constant $K$-invariant polynomials on the underlying real space $V_{\mathbb{R}}$ for $V$. These are nonholomorphic. The algebra $\mathbb{C}\left[V_{\mathbb{R}}\right]^{K}$ of such invariant polynomials has a natural vector space basis. To describe this, we introduce the Fock (or Fischer) inner product given on both $\mathbb{C}[V]$ and $\mathbb{C}\left[V_{\mathbb{R}}\right]$ by

$$
\langle f, g\rangle_{\mathscr{F}}=\left(\frac{1}{2 \pi}\right)^{n} \int_{V} f(z) \overline{g(z)} e^{-|z|^{2} / 2} d z
$$

Here $n=\operatorname{dim}_{\mathbb{C}}(V)$ and ' $d z$ ' denotes Lebesgue measure on $V_{\mathbb{R}} \cong \mathbb{R}^{2 n}$. For $\alpha \in \Lambda$ let $d_{\alpha}=\operatorname{dim}\left(P_{\alpha}\right)$, choose an orthonormal basis $\left\{v_{1}, v_{2}, \ldots, v_{d_{\alpha}}\right\}$ for $P_{\alpha}$, and set

$$
p_{\alpha}(z)=\frac{1}{d_{\alpha}} \sum_{j=1}^{d_{\alpha}} v_{j}(z) \overline{v_{j}(z)} .
$$

It is not difficult to see that this definition of the $p_{\alpha}$ 's does not depend on the choice of orthonormal basis for $P_{\alpha}$ and that $\left\{p_{\alpha} \mid \alpha \in \Lambda\right\}$ is a basis for the vector space $\mathbb{C}\left[V_{\mathbb{R}}\right]^{K}$ (see [2, Proposition 3.9]).

One obtains a second basis for $\mathbb{C}\left[V_{\mathbb{R}}\right]^{K}$ by orthogonalization of the $p_{\alpha}$ 's. More precisely, we

(1) choose any ordering on the set of indices $\Lambda$ that ensures $\alpha$ precedes $\beta$ if $|\alpha|<|\beta|$,

(2) use the Fock inner product and this ordering to perform Gram-Schmidt orthogonalization on the sequence $\left\{p_{\alpha} \mid \alpha \in \Lambda\right\}$, and

(3) normalize the resulting polynomials $q_{\alpha}$ so that $q_{\alpha}(0)=1$.

In [2, Proposition 4.2] we show that the resulting basis $\left\{q_{\alpha} \mid \alpha \in \Lambda\right\}$ does not depend on the ordering chosen for the indices $\{\alpha \in \Lambda|| \alpha \mid=m\}$ that arise from the decomposition of $\mathscr{P}_{m}(V), m \in \mathbb{Z}_{+}$. Our proof of this fact in [2] involves the representation theory of the Heisenberg group. We can suppress the Heisenberg 
group and outline the key ideas as follows. For $z \in V$ and $f \in \mathbb{C}[V]$ let $\pi(z) f$ be the function on $V$ given as

$$
\pi(z) f(w)=e^{-\langle w, z\rangle / 2-|z|^{2} / 4} f(w+z)
$$

and define functions $q_{\alpha}$ via

$$
q_{\alpha}(z)=\frac{1}{d_{\alpha}} \sum_{j=1}^{d_{\alpha}}\left\langle\pi(z) v_{j}, v_{j}\right\rangle_{\mathcal{F}}
$$

It is shown in [2] that

(1) $q_{\alpha}$ is a well defined $K$-invariant polynomial on $V_{\mathbf{R}}$ with $q_{\alpha}(0)=1$,

(2) the $q_{\alpha}$ 's are pair-wise orthogonal with respect to the Fock inner product, and

(3) the homogeneous component of highest degree in $q_{\alpha}$ is $(-1)^{|\alpha|} p_{\alpha}$.

It follows from these facts that if one chooses any ordering for $\Lambda$ as above then orthogonalization yields the polynomials $q_{\alpha}$ given by (1.4).

We now have two canonical bases $\left\{p_{\alpha} \mid \alpha \in \Lambda\right\}$ and $\left\{q_{\alpha} \mid \alpha \in \Lambda\right\}$ for $\mathbb{C}\left[V_{\mathbb{R}}\right]^{K}$. The $p_{\alpha}$ 's are homogeneous (of degree $2|\alpha|$ ) and the $q_{\alpha}$ 's are orthogonal. We write the $q_{\alpha}$ 's as linear combinations of the $p_{\alpha}$ 's:

$$
q_{\alpha}=\sum_{|\beta| \leq|\alpha|}(-1)^{|\beta|}\left[\begin{array}{l}
\alpha \\
\beta
\end{array}\right] p_{\beta} .
$$

Since the $p_{\alpha}$ 's and $q_{\alpha}$ 's are real valued functions, the coefficients $\left[\begin{array}{l}\alpha \\ \beta\end{array}\right]$ are real. We call these the generalized binomial coefficients for the multiplicity free action of $K$ on $V$. They are defined via (1.5) for $|\beta| \leq|\alpha|$. We extend the definition to all of $\Lambda \times \Lambda$ by setting $\left[\begin{array}{c}\alpha \\ \beta\end{array}\right]=0$ when $|\beta|>|\alpha|$.

The inclusion of the sign ' $(-1)^{|\beta|}$ ' in (1.5) is motivated by the fact that $(-1)^{|\alpha|} p_{\alpha}$ is the homogeneous component of highest degree in $q_{\alpha}$. Thus $\left[\begin{array}{l}\alpha \\ \alpha\end{array}\right]=1$ and $\left[\begin{array}{l}\alpha \\ \beta\end{array}\right]=0$ for $\alpha \neq \beta$ with $|\alpha|=|\beta|$. Moreover, Lemma 3.9 in [6] shows that, with this convention, the generalized binomial coefficients are non-negative. The main result in this paper asserts that these are, moreover, rational numbers.

THEOREM 1.1. $\left[\begin{array}{l}\alpha \\ \beta\end{array}\right]$ is a non-negative rational number for all $\alpha, \beta \in \Lambda$.

The generalized binomial coefficients were introduced in the setting of multiplicity free actions by Yan in his unpublished work [17]. The simplest example of a multiplicity free action is given by the standard action of $K=U(n)$ on $V=\mathbb{C}^{n}$. In this case $\mathbb{C}[V]$ decomposes as $\mathbb{C}[V]=\sum_{m} \mathscr{P}_{m}(V)$ and one can show (see [17] or [6]) that $\left[\begin{array}{l}m \\ j\end{array}\right]=\left(\begin{array}{l}m \\ j\end{array}\right)$, independent of $n$. This motivates the terminology. For multiplicity free actions that arise from Hermitian symmetric spaces, Yan has shown that the generalized binomial coefficients as defined above agree with those introduced by Herz, Dib 
and Faraut-Koranyi (see $[8,9,18])$. Our paper [6] shows that the generalized binomial coefficients appear in the solutions to a variety of combinatorial and analytic problems that arise in connection with multiplicity free actions and related Gelfand pairs associated with the Heisenberg group. We indicate one such interconnection. From $p_{\beta}$, one obtains a Wick ordered polynomial coefficient differential operator ' $p_{\beta}(z, \partial / \partial z)$ '. This is $K$-invariant and hence scalar on each subspace $P_{\alpha}$. The eigenvalue for this operator on $P_{\alpha}$ is $\left[\begin{array}{c}\alpha \\ \beta\end{array}\right] /\left(2^{|\beta|} d_{\beta}\right)$. This relates the computation of generalized binomial coefficients to that of eigenvalues for $K$-invariant polynomial coefficient differential operators, a problem that has been studied recently in [14-16]. Theorem 1.1 shows that these eigenvalues are non-negative rational numbers.

The rest of this paper is structured as follows. In Section 2 we show that the basis $\left\{p_{\alpha} \mid \alpha \in \Lambda\right\}$ contains a canonical subset $\left\{\gamma_{1}, \ldots, \gamma_{r}\right\}$ of 'fundamental invariants' that freely generate $\mathbb{C}\left[V_{\mathbb{R}}\right]^{K}$ as an algebra. We prove that one can replace the $p_{\alpha}$ 's by polynomials of the form $\gamma_{1}^{a_{1}} \cdots \gamma_{r}^{a_{r}}$ in the orthogonalization procedure used to determine the $q_{\alpha}$ 's. For this, we need to use a specific refinement of the partial ordering by degrees that is compatible with the weight ordering used to determine the fundamental invariants. This is the content of Theorem 2.1 below. In Section 3, we reduce the proof of Theorem 1.1 to the assertion that one can find some orthonormal basis for $V$ so that each fundamental invariant $\gamma_{i}$ is given by a polynomial with rational coefficients in the coordinates with respect to the basis. The proof uses Theorem 2.1 together with some results concerning generalized binomial coefficients from [6]. In Section 4 we complete the proof of Theorem 1.1 by showing the existence of such a basis. We do this via case-by-case analysis using a classification of multiplicity free actions and exhibiting rational fundamental highest weight vectors. This requires rather explicit knowledge of the decomposition for $\mathbb{C}[V]$ in each case. We believe that the details of this case-by-case analysis, which extends work of Howe and Umeda from [11], are of independent interest.

\section{Fundamental invariants}

Let $T$ be a maximal torus in $K$ and $G=K_{\mathrm{C}}, H=T_{\mathrm{C}}$ be the complexified groups with Lie algebras $\mathfrak{g}$ and $\mathfrak{h}$ respectively. Choose a positive system $\Delta^{+}=\Delta^{+}(\mathfrak{g}, \mathfrak{h})$ of roots. We recall that these choices produce a simple ordering on the weights $\lambda \in \mathfrak{h}^{*}=\operatorname{hom}(\mathfrak{h}, \mathfrak{C})$ for any representation of $K$. We denote this weight ordering by $\prec$. Let $\Lambda \subset \mathfrak{h}^{*}$ denote the set of highest weights for the irreducible representations of $K$ which occur in $\mathbb{C}[V]$. Thus $\Lambda$ is the index set for decomposition (1.1), and the irreducible component $P_{\alpha}$ has highest weight $\alpha \in \Lambda$.

If $h_{\alpha}, h_{\beta} \in \mathbb{C}[V]$ are $\alpha$ - and $\beta$-highest weight vectors, then $h_{\alpha} h_{\beta}$ is an $(\alpha+\beta)$ highest weight vector and thus $\Lambda \subset \mathfrak{h}^{*}$ is an additive semigroup. Following [11] we 
call the primitive elements of $\Lambda$ fundamental highest weights. These are the elements of $\Lambda$ that cannot be expressed as sums $\alpha+\beta$ with $\alpha, \beta \in \Lambda$. We see that if $h_{\alpha}$ is a prime polynomial, then $\alpha$ is a fundamental highest weight. As explained in [11], the converse is also true. The fundamental highest weights are finite in number and freely generate $\Lambda$. Let

$$
\left\{\alpha_{1}, \alpha_{2}, \ldots, \alpha_{r}\right\}
$$

be the fundamental highest weights listed in increasing order using $\prec$. We then have

$$
\Lambda=\left\{a_{1} \alpha_{1}+\cdots+a_{r} \alpha_{r} \mid a_{1}, \ldots, a_{r} \in \mathbb{Z}_{+}\right\}
$$

where $\mathbb{Z}_{+}$denotes the non-negative integers. The fundamental highest weights thus establish a semigroup isomorphism

$$
\left(\mathbb{Z}_{+}\right)^{r} \cong \Lambda, \quad\left(a_{1}, \ldots, a_{r}\right) \mapsto a_{1} \alpha_{1}+\cdots+a_{r} \alpha_{r} .
$$

This correspondence is canonical having chosen the data $T$ and $\Delta^{+}$. We sometimes write elements $\alpha \in \Lambda$ as ' $\alpha=\left(a_{1}, \ldots, a_{r}\right)$ ' to mean $\alpha=a_{1} \alpha_{1}+\cdots+a_{r} \alpha_{r}$. In this notation we have $\alpha_{1}=(1,0, \ldots, 0), \alpha_{2}=(0,1,0, \ldots, 0)$ and so on.

DEFINITION 2.1. The fundamental invariants $\gamma_{1}, \ldots, \gamma_{r}$ are defined as $\gamma_{j}=p_{\alpha_{j}}$ where as above $\alpha_{1}, \ldots, \alpha_{r} \in \Lambda$ are the fundamental highest weights.

For $\alpha=\left(a_{1}, \ldots, a_{r}\right) \in \Lambda$ let

$$
\gamma_{\alpha}=\gamma_{1}^{a_{1}} \gamma_{2}^{a_{2}} \cdots \gamma_{r}^{a_{r}}
$$

Note that $\gamma_{\alpha}$ is $K$-invariant and homogeneous of degree $2|\alpha|=\operatorname{deg}\left(p_{\alpha}\right)$. We know that the $q_{\alpha}$ 's can be obtained by orthogonalization of the $p_{\alpha}$ 's using any ordering compatible with degree. We show that the $q_{\alpha}$ 's can also be obtained by orthogonalization of the $\gamma_{\alpha}$ 's. For this, we need to use the weight ordering $\prec$ on $\Lambda$ to refine the partial ordering by degrees.

THEOREM 2.1. The polynomials $\left\{q_{\alpha} \mid \alpha \in \Lambda\right\}$ are obtained from the polynomials $\left\{\gamma_{\alpha} \mid \alpha \in \Lambda\right\}$ by using the Fock inner product to perform Gram-Schmidt orthogonalization and normalizing so that $q_{\alpha}(0)=1$. Here we impose the ordering $<$ on $\Lambda$ defined by

$$
\alpha<\beta \Leftrightarrow[(|\alpha|<|\beta|) \text { or }(|\alpha|=|\beta| \text { and } \alpha \prec \beta)] \text {. }
$$


We remark that, from a practical viewpoint, Theorem 2.1 substantially improves the orthogonalization result discussed in Section 1. In many examples, it is much easier to obtain explicit formulae for the fundamental invariants, which are finite in number, than it is to produce formulae for all of the $p_{\alpha}$ 's. In such cases, the orthogonalization procedure in Theorem 2.1 is concrete in the sense that the sequence of $\gamma_{\alpha}$ 's is explicit. We provide formulae for the fundamental invariants for many of the examples discussed below in Section 4. In [3], Theorem 2.1 was obtained in the context of one specific multiplicity free action. This is Example 4.1.2 below.

ProOF OF THEOREM 2.1. Given $\lambda \in \mathfrak{h}^{*}$, we write $W_{\lambda}$ for the $\lambda$-weight space in $\mathbb{C}[V], W_{\lambda}=\{f \in \mathbb{C}[V] \mid X \cdot f=\lambda(X) f \forall X \in \mathfrak{h}\}$ in $\mathbb{C}[V]$. Choose a highest weight vector $h_{j} \in P_{\alpha_{j}}$ for each of the fundamental highest weights $\alpha_{1}, \ldots, \alpha_{r}$. The $h_{j}$ 's are unique up to non-zero scalar multiples. We normalize to ensure $\left\langle h_{j}, h_{j}\right\rangle_{\mathscr{F}}=1$. Since $P_{\alpha_{j}}$ is an orthogonal direct sum of its weight spaces, we can write the fundamental invariant $\gamma_{j}=p_{\alpha_{j}}$ in the form

$$
\gamma_{j}=\frac{1}{d_{\alpha_{j}}} h_{j} \bar{h}_{j}+r_{j}
$$

where $r_{j} \in \sum_{\lambda<\alpha_{j}} W_{\lambda} \otimes \bar{W}_{\lambda}$. For $\alpha=\left(a_{1}, \ldots, a_{r}\right) \in \Lambda$ we let $h_{\alpha}=h_{1}^{a_{1}} h_{2}^{a_{2}} \cdots h_{r}^{a_{r}}$. This is a highest weight vector in $P_{\alpha}$. From (2.2) we see that

$$
\gamma_{\alpha}=\frac{1}{d_{\alpha_{1}}^{a_{1}} \cdots d_{\alpha_{r}}^{a_{r}}} h_{\alpha} \bar{h}_{\alpha}+r_{\alpha}
$$

where $r_{\alpha} \in \sum_{\lambda<\alpha} W_{\lambda} \otimes \bar{W}_{\lambda}$. Similarly, we see that $p_{\alpha}$ can also be written in the form

$$
p_{\alpha}=\frac{b_{\alpha}}{d_{\alpha}} h_{\alpha} \bar{h}_{\alpha}+r_{\alpha}^{\prime}
$$

where $b_{\alpha}=1 /\left\|h_{\alpha}\right\|^{2}$ is a positive constant and $r_{\alpha}^{\prime} \in \sum_{\lambda<\alpha} W_{\lambda} \otimes \bar{W}_{\lambda}$.

Comparing (2.3) and (2.4), we see that

$$
p_{\alpha}=c_{\alpha} \gamma_{\alpha}+s_{\alpha}
$$

where $c_{\alpha}$ is a positive constant and $s_{\alpha} \in \sum_{\lambda<\alpha} W_{\lambda} \otimes \bar{W}_{\lambda}$. As both $p_{\alpha}$ and $\gamma_{\alpha}$ are $K$-invariant, so is $s_{\alpha}$. Since $\left\{p_{\alpha} \mid \alpha \in \Lambda\right\}$ is a basis for $\mathbb{C}\left[V_{R}\right]^{K}$, we have that $s_{\alpha} \in \operatorname{Span}\left(p_{\beta} \mid \beta \in \Lambda, \beta \prec \alpha\right)$. As both $p_{\alpha}$ and $\gamma_{\alpha}$ are homogeneous of degree $2|\alpha|$, equation (2.5) also shows that $s_{\alpha}$ is homogeneous of degree $2|\alpha|$, provided $s_{\alpha} \neq 0$. Thus we must have

$$
s_{\alpha} \in \operatorname{Span}\left(p_{\beta}|| \beta|=| \alpha \mid \text { and } \beta<\alpha\right) \subset \operatorname{Span}\left(p_{\beta} \mid \beta<\alpha\right) .
$$


This fact together with (2.5) implies that application of Gram-Schmidt orthogonalization to the sequences $\left\{p_{\alpha} \mid \alpha \in \Lambda\right\}$ and $\left\{\gamma_{\alpha} \mid \alpha \in \Lambda\right\}$ ordered via $<$ yield the same result. Since we know that orthogonalization of the $p_{\alpha}$ 's yields the $q_{\alpha}$ 's, this completes the proof.

REMARK 2.1. We note that the proof of Theorem 2.1 shows that for fixed $m$ and $\alpha=\min \{\beta \in \Lambda|| \beta \mid=m\}$ one has

$$
p_{\alpha}=c_{\alpha} \gamma_{\alpha}
$$

where $c_{\alpha}=\left(d_{\alpha_{1}}^{a_{1}} \cdots d_{\alpha_{r}}^{a_{r}}\right) /\left(d_{\alpha}\left\|h_{\alpha}\right\|^{2}\right)$.

\section{Proof of Theorem 1.1}

Suppose that $\left\{e_{1}, \ldots, e_{n}\right\}$ is an orthonormal basis for the Hermitian vector space $V$. We obtain isomorphisms $V \cong \mathbb{C}^{n}$ and

$$
\mathbb{C}[V] \cong \mathbb{C}\left[z_{1}, \ldots, z_{n}\right], \quad \mathbb{C}\left[V_{\mathbb{R}}\right] \cong \mathbb{C}\left[z_{1}, \ldots, z_{n}, \bar{z}_{1}, \ldots, \bar{z}_{n}\right]
$$

where $z_{1}, \ldots, z_{n}$ are the coordinates with respect to the basis. We write $\mathbb{Q}[V]$ and $\mathbb{Q}\left[V_{\mathbb{R}}\right]$ for the $\mathbb{Q}$-subalgebras of $\mathbb{C}[V]$ and $\mathbb{C}\left[V_{\mathbb{R}}\right]$ that correspond to $\mathbb{Q}\left[z_{1}, \ldots, z_{n}\right]$ and $\mathbb{Q}\left[z_{1}, \ldots, z_{n}, \bar{z}_{1}, \ldots, \bar{z}_{n}\right]$ under these identifications. Our notation conceals the fact that $\mathbb{Q}[V]$ and $\mathbb{Q}\left[V_{\mathbb{R}}\right]$ depend on the orthonormal basis used. In Section 4 we prove the following result.

LEMMA 3.1. There is an orthonormal basis for $V$ in which $\gamma_{1}, \ldots, \gamma_{r} \in \mathbb{Q}\left[V_{\mathbb{R}}\right]$.

This result is a key ingredient in our proof of Theorem 1.1. We assume Lemma 3.1 here and complete the proof of Theorem 1.1. Fix an orthonormal basis for $V$ so that the fundamental invariants are rational polynomials as in Lemma 3.1.

Next we recall that the monomials $z^{I}:=z_{1}^{i_{1}} \cdots z_{n}^{i_{n}}$ in $\mathbb{C}[V] \cong \mathbb{C}\left[z_{1}, \ldots, z_{n}\right]$ are orthogonal with respect to $\langle\cdot, \cdot\rangle_{\xi}$ and satisfy $\left\langle z^{I}, z^{I}\right\rangle_{m}=2^{I} I !:=2^{i_{1}+\cdots+i_{n}} i_{1} ! \cdots i_{n} !$. (See for example [10, Theorem 1.63], although this reference uses a different normalization convention for the Fock inner product.) Thus we also have

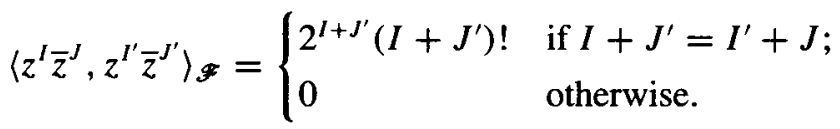

In particular, the inner product of any two monomials in $\mathbb{C}\left[V_{\mathbb{R}}\right]$ is integral. We conclude immediately that

LEMMA 3.2. $\langle f, g\rangle_{\xi} \in \mathbb{Q}$ for any $f, g \in \mathbb{Q}\left[V_{\mathbb{R}}\right]$. 
LEMMA 3.3. $q_{\alpha} \in \mathbb{Q}\left[V_{\mathbb{R}}\right]$ for all $\alpha \in \Lambda$.

ProOF. We use the ordering specified in Theorem 2.1 to perform Gram-Schmidt orthogonalization with the sequence of polynomials $\left\{\gamma_{\alpha} \mid \alpha \in \Lambda\right\}$. This yields an (unnormalized) sequence of orthogonal polynomials $\tilde{q}_{\alpha}$ with $\tilde{q}_{0}=1$ and

$$
\tilde{q}_{\alpha}=\gamma_{\alpha}-\sum_{\beta<\alpha} \frac{\left\langle\gamma_{\alpha}, \tilde{q}_{\beta}\right\rangle_{\mathscr{F}}}{\left\langle\widetilde{q}_{\beta}, \widetilde{q}_{\beta}\right\rangle_{\mathscr{F}}} \tilde{q}_{\beta}
$$

for $\alpha>0$. Here $0=(0, \ldots, 0) \in \Lambda$ is the index for which $P_{0}=\mathbb{C}$, the scalar polynomials. We have that $\widetilde{q}_{0} \in \mathbb{Q}\left[V_{\mathbb{R}}\right]$. Assume inductively that $\alpha>0$ and that $\tilde{q}_{\beta} \in \mathbb{Q}\left[V_{\mathbb{R}}\right]$ for $\beta<\alpha$. Lemma 3.1 shows that $\gamma_{\alpha} \in \mathbb{Q}\left[V_{\mathbb{R}}\right]$ and Lemma 3.2 ensures that the coefficients in the expression for $\tilde{q}_{\alpha}$ are all rational. Thus $\tilde{q}_{\alpha} \in \mathbb{Q}\left[V_{\mathbf{R}}\right]$ for all $\alpha \in \Lambda$. A second induction on $\alpha$ shows that $\widetilde{q}_{\alpha}(0) \in \mathbb{Q}$. Thus also $q_{\alpha}=\widetilde{q}_{\alpha} / \widetilde{q}_{\alpha}(0) \in$ $\mathbb{Q}\left[V_{\mathbb{R}}\right]$.

We can now complete the proof of Theorem 1.1 by using some combinatorial identities from [6]. Proposition 3.7 in that paper asserts that

$$
\frac{|z|^{2}}{2} q_{\beta}=-\sum_{|\alpha|=|\beta|+1} \frac{d_{\alpha}}{d_{\beta}}\left[\begin{array}{l}
\alpha \\
\beta
\end{array}\right] q_{\alpha}+(2|\beta|+n) q_{\beta}-\sum_{|\alpha|=|\beta|-1}\left[\begin{array}{l}
\beta \\
\alpha
\end{array}\right] q_{\alpha} .
$$

We know, moreover, that the $q_{\alpha}$ 's are orthogonal and the norms are given by $\left\langle q_{\alpha}, q_{\alpha}\right\rangle_{\mathcal{F}}=1 / d_{\alpha}$. (See $[4,17]$.) Thus, for $|\alpha|=|\beta|+1$ we have

$$
\left\langle\frac{|z|^{2}}{2} q_{\beta}, q_{\alpha}\right\rangle_{\xi}=-\frac{1}{d_{\beta}}\left[\begin{array}{l}
\alpha \\
\beta
\end{array}\right]
$$

Lemma 3.3 shows that $q_{\alpha}$ and $|z|^{2} q_{\beta} / 2$ belong to $\mathbb{Q}\left[V_{\mathbb{R}}\right]$ and hence the left hand side of the last equation is rational by Lemma 3.2. It follows that $\left[\begin{array}{l}\alpha \\ \beta\end{array}\right] \in \mathbb{Q}$ whenever $|\alpha|=|\beta|+1$. Suppose more generally that $|\alpha|=|\beta|+k$. Equation (3.9) in [6] reads:

$$
\left[\begin{array}{l}
\alpha \\
\beta
\end{array}\right]=\frac{1}{k !} \sum\left[\begin{array}{l}
\varepsilon_{1} \\
\beta
\end{array}\right]\left[\begin{array}{l}
\varepsilon_{2} \\
\varepsilon_{1}
\end{array}\right] \ldots\left[\begin{array}{c}
\varepsilon_{k-1} \\
\varepsilon_{k-2}
\end{array}\right]\left[\begin{array}{c}
\alpha \\
\varepsilon_{k-1}
\end{array}\right]
$$

where the sum is over all $\left(\varepsilon_{1}, \ldots, \varepsilon_{k-1}\right)$ with $\left|\varepsilon_{j}\right|=|\beta|+j$. Since all the generalized binomial coefficients appearing on the right hand side of this equation are rational, we conclude that $\left[\begin{array}{l}\alpha \\ \beta\end{array}\right] \in \mathbb{Q}$.

\section{Case-by-case analysis}

In this section we prove Lemma 3.1. This involves case-by-case analysis working from the classification of multiplicity free actions contained in [5]. We begin with a 
simple lemma that enables a substantial reduction in the work required for each case. First note that the concept of multiplicity free action depends only on the image of $K$ in $U(V)$ under its representation on $V$. Thus we can regard $K$ as a subgroup of $U(V)$ acting on $V$ in the usual fashion. Recall that we use an orthonormal basis of $V$ to write polynomials in coordinates and identify rational subalgebras $\mathbb{Q}[V]$ and $\mathbb{Q}\left[V_{\mathbb{R}}\right]$ of $\mathbb{C}[V]$ and $\mathbb{C}\left[V_{\mathbb{R}}\right]$. Such a basis can also be used to realize $U(V)$ and $G L(V)$ as the matrix groups $U(n)$ and $\mathrm{GL}(n, \mathbb{C})$. The group $K$ and its complexification $G=K_{\mathrm{C}}$ become subgroups of $\operatorname{GL}(n, \mathbb{C})$ acting on $\mathbb{C}^{n}$ in the standard fashion. The Lie algebra $\mathfrak{g}$ of $G$ becomes a subalgebra of the Lie algebra $g l(n, \mathbb{C})$ of $n \times n$ matrices. Let $\mathfrak{g}_{\mathbf{Q}}=\mathfrak{g} \cap g l(n, \mathbb{Q})$. This is a Lie algebra over $\mathbb{Q}$. One says that $\mathfrak{g}_{\mathbb{Q}}$ is a rational form for $\mathfrak{g}$ if $\mathfrak{g}=\mathbb{C} \otimes \mathfrak{g} \mathbf{Q}$. Equivalently, $\mathfrak{g}$ has a basis (over $\mathbb{C}$ ) that is contained in the subset $g_{\mathbf{Q}}$.

LEMMA 4.1. Suppose that $\mathrm{g}_{\mathrm{Q}}$ is a rational form for $\mathrm{g}$. Let $\alpha \in \Lambda$ and suppose that $P_{\alpha} \cap \mathbb{Q}[V] \neq\{0\}$. Then $p_{\alpha} \in \mathbb{Q}\left[V_{\mathbb{R}}\right]$.

PROOF. Let $h \in P_{\alpha} \cap \mathbb{Q}[V]$ with $h \neq 0$. Since $P_{\alpha}$ is $K$-irreducible we have $P_{\alpha}=\mathscr{U}(\mathfrak{g}) h$. Thus any basis $\left\{f_{1}, \ldots, f_{m}\right\}$ for $P_{\alpha}\left(m=d_{\alpha}\right)$ can be written as

$$
f_{1}=D_{1} h, f_{2}=D_{2} h, \ldots, f_{m}=D_{m} h
$$

for some $D_{1}, \ldots, D_{m} \in \mathscr{U}(\mathfrak{g})$. Since $\mathscr{U}(\mathfrak{g})=\mathscr{U}\left(\mathbb{C} \otimes \mathfrak{g}_{\mathfrak{Q}}\right)=\mathbb{C} \otimes \mathscr{U}\left(\mathfrak{g}_{\mathbf{Q}}\right)$, we can write

$$
D_{j}=c_{j, 1} D_{j, 1}+\cdots+c_{j, \ell_{j}} D_{j, \ell_{j}}
$$

for some $c_{j, 1}, \ldots, c_{j, \ell_{j}} \in \mathbb{C}$ and $D_{j, 1}, \ldots, D_{j, \ell_{j}} \in \mathscr{U}\left(\mathrm{g}_{\mathbf{Q}}\right)$. Thus

$$
\begin{aligned}
P_{\alpha} & =\mathbb{C}-\operatorname{Span}\left(f_{1}, \ldots, f_{m}\right) \\
& =\mathbb{C}-\operatorname{Span}\left(D_{1,1} h, \ldots, D_{1, \ell_{1}} h, D_{2,1} h, \ldots, D_{2, \ell_{2}} h, \ldots, D_{m, 1} h, \ldots, D_{m, \ell_{m}} h\right)
\end{aligned}
$$

and we conclude that $P_{\alpha}$ has a basis consisting of vectors of the form $D_{i, j} h$. Let $\left\{g_{1}, \ldots, g_{m}\right\}$ denote such a basis. Since $D_{i, j} \in \mathscr{U}\left(g_{\mathbb{Q}}\right) \subset \mathscr{U}(g l(n, \mathbb{Q}))$ and $g l(n, \mathbb{Q})$ preserves $\mathbb{Q}[V]=\mathbb{Q}\left[z_{1}, \ldots, z_{n}\right]$, we have that $g_{1}, \ldots, g_{m} \in \mathbb{Q}[V]$.

Next we orthogonalize the basis $\left\{g_{1}, \ldots, g_{m}\right\}$ to obtain a basis $\left\{u_{1}, \ldots, u_{m}\right\}$ defined as $u_{1}=g_{1}$ and

$$
u_{j}=g_{j}-\sum_{i=1}^{j-1} \frac{\left\langle g_{j}, u_{i}\right\rangle_{\mathscr{F}}}{\left\langle u_{i}, u_{i}\right\rangle_{\mathscr{F}}} u_{i}
$$

for $i=2, \ldots, m$. Since the $g_{j}$ 's belong to $\mathbb{Q}[V]$, we can use Lemma 3.2 and induction, as in the proof for Lemma 3.3, to conclude that $u_{j} \in \mathbb{Q}[V]$. Since $\left\{u_{1}, \ldots, u_{m}\right\}$ is an 
orthogonal basis for $P_{\alpha}$, we can use $v_{j}=u_{j} /\left\|u_{j}\right\|$ in (1.3) to write

$$
p_{\alpha}(z)=\frac{1}{m} \sum_{j=1}^{m} \frac{u_{j}(z) \overline{u_{j}(z)}}{\left\langle u_{j}, u_{j}\right\rangle_{F}} .
$$

Here $\left\langle u_{j}, u_{j}\right\rangle_{\mathcal{F}} \in \mathbb{Q}$ since $u_{j} \in \mathbb{Q}[V]$. Thus we see that $p_{\alpha} \in \mathbb{Q}[V]$.

To prove Lemma 3.1, it now suffices to show that for any multiplicity free action:

(1) there is an orthonormal basis for $V$ for which $\mathfrak{g}_{\mathbb{Q}}=\mathfrak{g} \cap g l(n, \mathbb{Q})$ is a rational form for $\mathfrak{g}$, and

(2) one can find fundamental highest weight vectors $h_{j} \in P_{\alpha_{j}}(j=1, \ldots, r)$ that are rational polynomials in the coordinates with respect to this basis. That is, $h_{j} \in \mathbb{Q}[V]$ for $j=1, \ldots, r$.

This completes the proof. Indeed, Lemma 4.1 shows that using such a basis we have $\gamma_{1}, \ldots, \gamma_{r} \in \mathbb{Q}\left[V_{\mathbb{R}}\right]$.

The group $G \subset \mathrm{GL}(V)$ is connected reductive and complex algebraic. Decompose $V$ as an orthogonal direct sum of $G$-irreducible subspaces:

$$
V=V_{1} \oplus \cdots \oplus V_{m} .
$$

For our purposes, we can replace $G$ by $\widetilde{G}=G^{\prime} \times\left(\mathbb{C}^{\times}\right)^{m}$ where $G^{\prime}$ denotes the commutator subgroup and we have one copy of the scalars $\mathbb{C}^{\times}$acting on each subspace $V_{j}$. Indeed, the decompositions of $\mathbb{C}[V]$ under the actions of $G$ and $\widetilde{G}$ coincide and hence the $p_{\alpha}$ 's, $q_{\alpha}$ 's and generalized binomial coefficients for the two actions are the same. We can assume, moreover, that the action of the semisimple group $G^{\prime}$ on $V$ is indecomposable. This means that we can't write $V$ as a direct sum $V=W_{1} \oplus W_{2}$ and $G^{\prime}$ as a product $G^{\prime}=G_{1}^{\prime} \times G_{2}^{\prime}$ with $G_{j}^{\prime}$ acting independently on $W_{j}$. Indeed, suppose that given any such indecomposable action one can find an orthonormal basis with respect to which the fundamental invariants are rational polynomials. If the action of $G^{\prime}$ decomposes as a product of indecomposable actions of subgroups $G_{j}^{\prime}$ on subspaces $W_{j}$ then one obtains an orthonormal basis for $V$ that meets our requirements by concatenation of appropriately chosen bases for the $W_{j}$ 's. In particular, these bases produce obvious inclusions $\mathbb{Q}\left[\left(W_{j}\right)_{\mathbb{R}}\right] \subset \mathbb{Q}\left[V_{\mathbb{R}}\right]$ and the fundamental invariants $\gamma_{j, i} \in \mathbb{Q}\left[\left(W_{j}\right)_{\mathbb{R}}\right]$ for the actions of each $G_{j}^{\prime} \times\left(\mathbb{C}^{\times}\right)^{m_{j}}$ on $W_{j}$ just combine to yield the (rational) fundamental invariants for the action of $\widetilde{G}$.

We can now simplify the notation from the preceding paragraph and restrict our attention to actions of the following sort.

- $G \subset \mathrm{GL}(V)$ is a connected semisimple complex algebraic group acting indecomposably on $V$.

- $V=V_{1} \oplus \cdots \oplus V_{m}$, where each $V_{j}$ is $G$-irreducible.

- The joint action of $G \times\left(\mathbb{C}^{\times}\right)^{m}$ on $V$ is multiplicity free. 
TABLE 1.

\begin{tabular}{|l|l|l|}
\hline Group & $\begin{array}{l}\text { Degrees of fundamental highest } \\
\text { weight vectors (number) }\end{array}$ & $\begin{array}{l}\text { Example } \\
\text { number }\end{array}$ \\
\hline $\mathbf{S L}(\mathbf{n})(n \geq 1)$ & $1(1)$ & 4.1 .1 \\
$\mathbf{S O}(\mathbf{n})(n \geq 3)$ & $1,2(2)$ & 4.1 .2 \\
$\mathbf{S p}(\mathbf{2 n})(n \geq 2)$ & $1(1)$ & 4.1 .1 \\
$\mathbf{S}^{2}(\mathbf{S L}(\mathbf{n}))(n \geq 2)$ & $1,2, \ldots, n(n)$ & 4.1 .4 \\
$\Lambda^{2}(\mathbf{S L}(\mathbf{n}))(n \geq 4)$ & $1,2, \ldots,\lfloor n / 2\rfloor(\lfloor n / 2\rfloor)$ & 4.1 .5 \\
$\mathbf{S L}(\mathbf{n}) \otimes \mathbf{S L}(\mathbf{m})(n, m \geq 2)$ & $1,2, \ldots, \min (n, m)(\min (n, m))$ & 4.1 .3 \\
$\mathbf{S p}(\mathbf{2 n}) \otimes \mathbf{S L}(\mathbf{2})(n \geq 2)$ & $1,2,2(3)$ & 4.1 .6 \\
$\mathbf{S p}(\mathbf{2 n}) \otimes \mathbf{S L}(\mathbf{3})(n \geq 2)$ & $1,2,2,3,3,4(6)$ & 4.1 .7 \\
$\mathbf{S p}(\mathbf{4}) \otimes \mathbf{S L}(\mathbf{n})(n \geq 4)$ & $1,2,2,3,4,4(6)$ & 4.1 .8 \\
$\mathbf{S p i n}(7)$ & $1,2(2)$ & 4.1 .9 \\
$\mathbf{S p i n}(\mathbf{9})$ & $1,2,2(3)$ & 4.1 .10 \\
$\mathbf{S p i n}(\mathbf{1 0})$ & $1,2(2)$ & 4.1 .11 \\
$\mathbf{G}_{\mathbf{2}}$ & $1,2(2)$ & 4.1 .2 \\
$\mathbf{E}_{\mathbf{6}}$ & $1,2,3(3)$ & 4.1 .12 \\
\hline
\end{tabular}

Such actions have been completely classified. In all cases, one has either $m=1$, so that the action of $G$ on $V$ is irreducible, or $m=2$. The irreducible multiplicity free actions were classified by Kac in [12]. The indecomposable non-irreducible actions were classified by the authors in [5] and independently by Leahy in [13]. Below, we examine each possibility in turn to complete the proof of Lemma 3.1.

4.1. Irreducible multiplicity free actions Table 1, taken from [12], lists all possibilities for semisimple groups $G \subset \mathrm{GL}(V)$ acting irreducibly on $V$ for which the action of $G \times\left(\mathbb{C}^{\times}\right)$is multiplicity free. The notation, adopted from [12], indicates $G$ as the image of a group under some irreducible representation on some vector space $V$. For example, $\Lambda^{2}(\mathbf{S L}(\mathbf{n}))$ indicates the image of $\operatorname{SL}(n, \mathbb{C})$ in $\operatorname{GL}\left(\Lambda^{2}\left(\mathbb{C}^{n}\right)\right)$ and $\operatorname{Sp}(2 n) \otimes \operatorname{SL}(3)$ indicates the image of $\operatorname{Sp}(2 n, \mathbb{C}) \times \operatorname{SL}(3, \mathbb{C})$ in $\mathrm{GL}\left(\mathbb{C}^{2 n} \otimes \mathbb{C}^{3}\right)$ under the obvious representations.

For all of the groups $G \subset \mathrm{GL}(V)$ in Table 1 , the vector space $V$ has a standard basis that is orthonormal for a natural Hermitian inner product that determines the compact real form $K \subset U(V)$. For the classical groups, these are the natural bases for $\mathbb{C}^{n}, \Lambda^{2}\left(\mathbb{C}^{n}\right), S^{2}\left(\mathbb{C}^{n}\right)$ and $\mathbb{C}^{n} \otimes \mathbb{C}^{m}$. Using these bases to realize $G$ as a matrix group $G \subset \mathrm{GL}(n, \mathbb{C})$, one sees easily that $\mathfrak{g}_{\mathbb{Q}}=\mathfrak{g} \cap g l(n, \mathbb{Q})$ is a rational form for $\mathfrak{g}$ in each case. Thus, it remains to show that one can find fundamental highest weight vectors that are rational polynomials in the coordinates with respect to these natural bases. We do this below in each case. The fundamental highest weight vectors for 
most of these actions were given explicitly by Howe and Umeda in [11]. In all cases, the number of fundamental highest weight vectors and their degrees (as homogeneous polynomials in $\mathbb{C}[V])$ are in [11]. We have incorporated this information into Table 1. In many cases, we are able to present explicit formulae for the fundamental invariants, at least up to scalar multiples. We follow the notational conventions in [11], to which we refer the reader for further details regarding the decomposition of $\mathbb{C}[V]$ for each of these examples.

4.1.1. $\operatorname{SL}(\mathbf{n}), \operatorname{Sp}(2 \mathrm{n}) \quad$ Here $G=\operatorname{SL}(n, \mathbb{C})$ and $G=\operatorname{Sp}(2 n, \mathbb{C})$ act on $V=\mathbb{C}^{n}$ and $V=\mathbb{C}^{2 n}$ by their defining representations. The $G$-irreducible subspaces of $\mathbb{C}[V]$ are the spaces $\mathscr{P}_{m}(V)$ of homogeneous polynomials of each fixed degree $m$. There is a single fundamental highest weight vector, $z_{1} \in \mathscr{P}_{1}(V)$ and the associated fundamental invariant is $\gamma(z)=|z|^{2} / 2$. As we have already noted, the generalized binomial coefficients for these examples are the usual binomial coefficients, motivating our terminology.

4.1.2. $\mathbf{S O}(\mathbf{n}), \mathbf{G}_{2} \quad$ Let $\varepsilon(z)=z_{1}^{2}+\cdots+z_{n}^{2}$ be the $\operatorname{SO}(n, \mathbb{C})$-invariant polynomial on $V=\mathbb{C}^{n}$ and let

$$
D_{\varepsilon}=\left(\frac{\partial}{\partial z_{1}}\right)^{2}+\cdots+\left(\frac{\partial}{\partial z_{n}}\right)^{2} .
$$

Then $\mathscr{H}=\left\{p \in \mathbb{C}[V] \mid D_{\varepsilon} p=0\right\}$ is the space of 'harmonics', with $\mathscr{H}=\sum_{m=0}^{\infty} \mathscr{H}_{m}$, $\mathscr{H}_{m}=\mathscr{H} \cap \mathscr{P}_{m}(V)$. The decomposition of $\mathbb{C}[V]$ into $\mathrm{SO}(n, \mathbb{C}) \times \mathbb{C}^{\times}$-irreducibles reads:

$$
\mathbb{C}[V]=\sum_{m, \ell} \mathscr{H}_{m} \varepsilon^{\ell}
$$

There are two fundamental highest weight vectors in $\mathbb{C}[V], z_{1}$ and $\varepsilon(z)$. The associated fundamental invariants are $\gamma_{1}(z)=|z|^{2} / 2$ and $\gamma_{2}(z)=|\varepsilon(z)|^{2} / 4 n$. For further details on this example, we refer the reader to [3].

The exceptional group $G_{2}$ acts on $V=\mathbb{C}^{7}$ as a subgroup of $\operatorname{SO}(7, \mathbb{C})$. The subspaces $\mathscr{H}_{m} \varepsilon^{\ell}$ in $\mathbb{C}[V]$ are irreducible for the action of $G_{2} \times \mathbb{C}^{\times}$. Thus the decomposition, fundamental highest weight vectors and invariants for this example are the same as those for $\mathbf{S O}(7)$.

In the following examples, $V$ is $\mathbb{C}^{n} \otimes \mathbb{C}^{m}, \Lambda^{2}\left(\mathbb{C}^{n}\right), S^{2}\left(\mathbb{C}^{n}\right)$ or $\mathbb{C}^{n} \oplus \mathbb{C}^{n}$. We regard $V$ as the space of $n \times m$ matrices, skew-symmetric $n \times n$ matrices, symmetric $n \times n$ matrices or $n \times 2$ matrices respectively. We write $z=\left(z_{i j}\right)$ for the coordinates of $z \in V$ with respect to the standard basis for $V$ and use the notation

$$
\operatorname{det}_{k}(z)=\operatorname{det}\left(\begin{array}{ccc}
z_{11} & \cdots & z_{1 k} \\
\vdots & \ddots & \vdots \\
z_{k 1} & \cdots & z_{k k}
\end{array}\right)
$$


4.1.3. $\mathbf{S L}(\mathbf{n}) \otimes \mathbf{S L}(\mathbf{m}) \quad$ The decomposition of $\mathbb{C}\left[\mathbb{C}^{n} \otimes \mathbb{C}^{m}\right]$ under the action of $\operatorname{SL}(n, \mathbb{C}) \times \operatorname{SL}(m, \mathbb{C}) \times \mathbb{C}^{\times}$is given by

$$
\mathbb{C}\left[\mathbb{C}^{n} \otimes \mathbb{C}^{m}\right]=\sum_{D} \rho_{D}^{n} \otimes \rho_{D}^{m}
$$

Here the sum is taken over all Young's diagrams with at most $\min (n, m)$ rows and $\rho_{D}^{n}, \rho_{D}^{m}$ are the representations of $\operatorname{SL}(n, \mathbb{C})$ and $\operatorname{SL}(m, \mathbb{C})$ corresponding to $D$. The fundamental highest weight vectors are

$$
\operatorname{det}_{k}(z) \quad \text { for } k=1, \ldots, \min (n, m) .
$$

The fundamental invariants can be written up to normalization as

$$
\gamma_{k}(z)=\sum_{|I|=|J|=k}\left|\operatorname{det}_{I, J}(z)\right|^{2} .
$$

Here $I, J$ denote subsets of $\{1, \ldots, n\}$ and $\{1, \ldots, m\}$ and $\operatorname{det}_{l, J}(z)$ is the subdeterminant of $z$ obtained from rows $I$ and columns $J$.

4.1.4. $\mathbf{S}^{2}(\mathbf{S L}(\mathbf{n})) \quad$ Here $\mathbb{C}\left[S^{2}\left(\mathbb{C}^{n}\right)\right]$ decomposes under the action of $\operatorname{SL}(n, \mathbb{C}) \times \mathbb{C}^{\times}$ as $\mathbb{C}\left[S^{2}\left(\mathbb{C}^{n}\right)\right]=\sum_{D} \rho_{D}^{n}$. The sum is over all Young's diagrams $D$ with at most $n$ rows, all of even length. The fundamental highest weight vectors $\operatorname{are} \operatorname{det}_{k}(z)$ for $k=1, \ldots, n$.

4.1.5. $\Lambda^{2}(\mathbf{S L}(\mathbf{n}))$ The decomposition is $\mathbb{C}\left[\Lambda^{2}\left(\mathbb{C}^{n}\right)\right]=\sum_{D} \rho_{D}^{n}$, where the sum is over all Young's diagrams $D$ with at most $n$ rows and each column is of even length. The fundamental highest weight vectors are $\zeta_{k}(z)$ for $k=1, \ldots,\lfloor n / 2\rfloor$, where $\zeta_{k}^{2}(z)=\operatorname{det}_{2 k}(z)$. That is, $\zeta_{k}(z)$ is the Pfaffian of the skew symmetric matrix given by the first $2 k$ rows and columns of $z$. This is written explicitly in [11] as

$$
\zeta_{k}(z)=\sum_{\sigma \in S_{2 k} / B_{k}} \operatorname{sign}(\sigma) z_{\sigma(1) \sigma(2)} \cdots z_{\sigma(2 k-1) \sigma(2 k)},
$$

where $B_{k}$ is the subgroup of the symmetric group $S_{2 k}$ which preserves the pairs $\{\{1,2\}, \ldots,\{2 k-1,2 k\}\}$. In particular, we see that $\zeta_{k}(z)$ is a rational polynomial in the entries of $z$. Let $I=\left\{i_{1}, \ldots, i_{2 k}\right\} \subset\{1, \ldots, n\}$ and let $\zeta_{I}$ be the Pfaffian computed with rows $I$ and columns $I$. Up to normalization, the fundamental invariants are $\gamma_{k}(z)=\sum_{|I|=2 k} \zeta_{I}(z) \zeta_{I}(\bar{z})$.

4.1.6. Sp(2n) $\otimes \mathbf{S L}(2) \quad$ Let $v_{i} \in \mathbb{C}^{2 n}$ be the columns of $z \in V=\mathbb{C}^{2 n} \otimes \mathbb{C}^{2}, i=1,2$. The symplectic product $\varepsilon(z)=\omega\left(v_{1}, v_{2}\right)$ of the columns of $z$ is an $\operatorname{Sp}(2 n, \mathbb{C})$-invariant. Let $D_{\varepsilon}=\varepsilon(\partial / \partial z)$, and $\mathscr{H}=\left\{p \in \mathbb{C}[V] \mid D_{\varepsilon} p=0\right\}$. We have

$$
\mathbb{C}[V]=\sum_{\alpha_{1} \geq \alpha_{2} \geq 0} \mathscr{H}_{\alpha_{1}, \alpha_{2}} \varepsilon^{\ell},
$$


where the irreducible subspace $\mathscr{H}_{\alpha_{1}, \alpha_{2}} \varepsilon^{\ell}$ has highest weight vector $z_{11}^{\alpha_{1}-\alpha_{2}} \operatorname{det}_{2}(z)^{\alpha_{2}} \varepsilon^{\ell}$. Thus the fundamental highest weight vectors are $z_{11}, \operatorname{det}_{2}(z)$ and $\varepsilon(z)$ and we have fundamental invariants $\gamma_{1}(z)=|z|^{2}$ and $\gamma_{2}(z)=|\varepsilon(z)|^{2}$, modulo normalizations. In order to compute the third fundamental invariant, we look at the decomposition of $\mathscr{P}_{2}(V)$. Under the action of $\mathrm{GL}(2 n, \mathbb{C}) \times \mathrm{GL}(2, \mathbb{C})$ we have

$$
\mathscr{P}_{2}(V)=\left(\rho_{(2)}^{2 n} \otimes \rho_{(2)}^{2}\right) \oplus\left(\rho_{(1,1)}^{2 n} \otimes \rho_{(1,1)}^{2}\right) .
$$

When we restrict to $\operatorname{Sp}(2 n, \mathbb{C}) \times \operatorname{GL}(2, \mathbb{C})$, the second component decomposes as $\mathscr{H}_{1,1} \oplus \mathbb{C} \varepsilon$. As we saw in Example 4.1.3, the (unnormalized) fundamental invariant corresponding to the second component in (4.2) is $\sum_{|I|=2} \operatorname{det}_{l}(z) \operatorname{det}_{l}(\bar{z})$, where $I$ indicates the choice of rows. Thus, if $\gamma_{2}^{\prime}$ is the invariant corresponding to $\mathscr{H}_{1,1}$, we can average over orthonormal bases for $\mathscr{H}_{1,1} \oplus \mathbb{C} \varepsilon$ in two different ways to obtain $\sum_{|I|=2}(1 / 8) \operatorname{det}_{I}(z) \operatorname{det}_{I}(\bar{z})=\gamma_{2}^{\prime}(z)+|\varepsilon(z)|^{2} /(8 n)$, so that

$$
\gamma_{2}^{\prime}(z)=\frac{1}{8}\left[\sum_{|l|=2} \operatorname{det}_{I}(z) \operatorname{det}_{l}(\bar{z})-\frac{|\varepsilon(z)|^{2}}{n}\right] .
$$

4.1.7. $\quad \mathbf{S p}(2 \mathrm{n}) \otimes \mathbf{S L}(3) \quad$ Again, we write the columns of $z \in \mathbb{C}^{2 n} \otimes \mathbb{C}^{3}$ as $v_{i} \in \mathbb{C}^{2 n}, i=1,2,3$. We have the three $\operatorname{Sp}(2 n, \mathbb{C})$-invariants $\varepsilon_{i j}(z)=\omega\left(v_{i}, v_{j}\right)$, $1 \leq i<j \leq 3$, and corresponding operators $D_{i j}=\varepsilon_{i j}(\partial / \partial z)$. The space of harmonic polynomials is $\mathscr{H}=\left\{p \in \mathbb{C}[V] \mid D_{i j} p=0\right.$ for all $\left.i, j\right\}$, which decomposes as $\mathscr{H}=$ $\sum \mathscr{H}_{\alpha_{1}, \alpha_{2}, \alpha_{3}}$, where $\mathscr{H}_{\alpha_{1}, \alpha_{2}, \alpha_{3}}$ has highest weight vector $z_{11}^{\alpha_{1}-\alpha_{2}} \operatorname{det}_{2}(z)^{\alpha_{2}-\alpha_{3}} \operatorname{det}_{3}(z)^{\alpha_{3}}$. The full decomposition of $\mathbb{C}[V]$ is (from [11]):

$$
\mathbb{C}[V]=\sum_{\substack{0 \leq b_{1} \leq \alpha_{1}-\alpha_{2} \\ 0 \leq b_{2} \leq \alpha_{2}-\alpha_{3} \\ b_{3} \geq 0}} \sigma_{\alpha_{1}, \alpha_{2}, \alpha_{3}}^{n} \otimes \rho_{\left(\alpha_{1}+b_{2}+b_{3}, b_{1}+\alpha_{2}+b_{3}, b_{1}+b_{2}+\alpha_{3}\right)}^{3},
$$

where $\sigma_{\alpha_{1}, \alpha_{2}, \alpha_{3}}^{n}$ is the representation of $\operatorname{Sp}(2 n, \mathbb{C})$ on $\mathscr{H}_{\alpha_{1}, \alpha_{2}, \alpha_{3}}$ and $\rho_{D}^{3}$ is the representation of $\operatorname{SL}(3, \mathbb{C})$ with Young's diagram $D$. By [11], we have six fundamental highest weight vectors:

$$
\begin{array}{lll}
\zeta_{1}=z_{11}, & \zeta_{2}^{\prime}=\varepsilon_{12}(z), & \zeta_{3}^{\prime}=z_{11} \varepsilon_{23}(z)-z_{12} \varepsilon_{13}(z)+z_{13} \varepsilon_{12}(z), \\
\zeta_{2}=\operatorname{det}_{2}(z), & \zeta_{3}=\operatorname{det}_{3}(z), & \zeta_{4}^{\prime}=\operatorname{det}_{12,13}(z) \varepsilon_{12}(z)-\operatorname{det}_{2}(z) \varepsilon_{13}(z) .
\end{array}
$$

We have the usual fundamental invariant $\gamma_{1}(z)=|z|^{2}$. The second degree fundamental invariants $\gamma_{2}(z)$ and $\gamma_{2}^{\prime}(z)$ are found in the components $\sigma_{1,1}^{n} \otimes \rho_{(1,1)}^{3}$ and $\sigma_{0}^{n} \otimes \rho_{(1,1)}^{3}$ respectively. The sum of these two components is the $\operatorname{SL}(2 n, \mathbb{C}) \times \operatorname{SL}(3, \mathbb{C})$ irreducible $\rho_{(1,1)}^{2 n} \otimes \rho_{(1,1)}^{3}$. As in the previous example, we take diagonal sums in two ways to obtain

$$
\frac{1}{8} \sum_{|I|=|J|=2} \operatorname{det}_{I, J}(z) \operatorname{det}_{I, J}(\bar{z})=\gamma_{2}(z)+\gamma_{2}^{\prime}(z) .
$$


On the other hand, $\rho_{0}^{2 n} \otimes \rho_{(1,1)}^{3}$ is spanned by the $\varepsilon_{i j}$ 's, and hence:

$$
\gamma_{2}^{\prime}(z)=\frac{1}{8 n} \sum \varepsilon_{i j}(z) \varepsilon_{i j}(\bar{z}), \quad \gamma_{2}(z)=\frac{1}{8} \sum_{|I|=|J|=2} \operatorname{det}_{I, J}(z) \operatorname{det}_{I, J}(\bar{z})-\frac{1}{8 n} \gamma_{2}^{\prime}(z) .
$$

The highest weight vector $\zeta_{3}^{\prime}$ lies in the the irreducible subspace $\sigma_{1,0,0}^{n} \otimes \rho_{(1,1,1)}^{3}$, which has dimension six. An orthogonal basis is given by $u_{i}^{\prime}=z_{i 1} \varepsilon_{23}-z_{i 2} \varepsilon_{13}+z_{i 3} \varepsilon_{12}$, for $i=1, \ldots, 6$. The first of these basis elements can be rewritten as

$$
u_{1}^{\prime}(z)=\operatorname{det}\left(\begin{array}{ccc}
z_{11} & z_{12} & z_{13} \\
z_{21} & z_{22} & z_{23} \\
z_{n+2,1} & z_{n+2,2} & z_{n+2,3}
\end{array}\right)+\operatorname{det}\left(\begin{array}{ccc}
z_{11} & z_{12} & z_{13} \\
z_{31} & z_{32} & z_{33} \\
z_{n+3,1} & z_{n+3,2} & z_{n+3,3}
\end{array}\right),
$$

which is a sum of twelve distinct monomials, each of norm 8 . Thus we obtain:

$$
\gamma_{3}^{\prime}(z)=\frac{1}{96} \sum_{i=1}^{6} u_{i}^{\prime}(z) u_{i}^{\prime}(\bar{z})
$$

The sum of subspaces $\left(\sigma_{1,1,1}^{n} \otimes \rho_{(1,1,1)}^{3}\right) \oplus\left(\sigma_{1,0,0} \otimes \rho_{(1,1,1)}^{3}\right)$ is the $\operatorname{SL}(2 n, \mathbb{C}) \times \operatorname{SL}(3, \mathbb{C})$ irreducible $\rho_{(1,1,1)}^{2 n} \otimes \rho_{(1,1,1)}^{3}$, and hence

$$
\gamma_{3}(z)=\frac{1}{48} \sum_{|I|=3} \operatorname{det}_{I}(z) \operatorname{det}_{I}(\bar{z})-\gamma_{3}^{\prime}(z)
$$

where the subscript $I$ indicates a choice of rows. We have not derived a formula for the fundamental invariant $\gamma_{4}^{\prime}$, which seems quite complicated.

4.1.8. Sp(4) $\otimes S L(n) \quad$ The fundamental highest weight vectors are, from [11],

$$
\begin{array}{lll}
\zeta_{1}=z_{11}, & \zeta_{2}=\operatorname{det}_{2}(z), & \zeta_{3}=\operatorname{det}_{3}(z), \\
\zeta_{4}=\operatorname{det}_{4}(z), & \zeta_{2}^{\prime}=\varepsilon_{12}(z), & \zeta_{4}^{\prime}=\operatorname{det}_{12,13}(z) \varepsilon_{12}(z)-\operatorname{det}_{2}(z) \varepsilon_{13}(z) .
\end{array}
$$

The highest weight vector $\zeta_{2}^{\prime}$ determines the space spanned by $\varepsilon_{i j}(z)=\omega\left(v_{i}, v_{j}\right)$, $1 \leq i<j \leq m$, and thus

$$
\gamma_{2}^{\prime}(z)=\frac{1}{16} \sum\left|\varepsilon_{i j}(z)\right|^{2} .
$$

As before, the spaces with highest weight vectors $\zeta_{2}$ and $\zeta_{2}^{\prime}$ sum to the $\operatorname{SL}(4, \mathbb{C}) \times$ $\operatorname{SL}(n, \mathbb{C})$-irreducible $\rho_{(1,1)}^{4} \otimes \rho_{(1,1)}^{m}$, and hence

$$
\gamma_{2}(z)=\frac{1}{48} \sum_{|I|=|J|=2}\left|\operatorname{det}_{I, J}(z)\right|^{2}-\gamma_{2}^{\prime}(z) \text {. }
$$


In degree three, the irreducible subspace containing $\zeta_{3}$ is the $\operatorname{SL}(4, \mathbb{C}) \times \operatorname{SL}(n, \mathbb{C})$ irreducible $\rho_{(1,1,1)}^{4} \otimes \rho_{(1,1,1)}^{n}$, and hence

Similarly,

$$
\gamma_{3}(z)=\frac{1}{8} \sum_{|I|=|J|=3}\left|\operatorname{det}_{I, J}(z)\right|^{2} .
$$

$$
\gamma_{4}(z)=\frac{1}{24 \cdot 16} \sum_{|J|=4}\left|\operatorname{det}_{J}(z)\right|^{2},
$$

where $J$ indicates a choice of columns.

4.1.9. $\operatorname{Spin}(7)$ The action here is the spin representation of $\operatorname{Spin}(7, \mathbb{C})$ on $V=$ $\Lambda\left(\mathbb{C}^{3}\right)$. We use the usual basis $1, e_{i}, e_{i} \wedge e_{j}, e_{1} \wedge e_{2} \wedge e_{3}$ for $V$ and let $f_{0}, f_{i}, f_{i j}, f_{123}$ respectively be the dual basis for $V^{*}$. The polynomial $\varepsilon=f_{0} f_{123}-f_{1} f_{23}+f_{2} f_{13}-f_{3} f_{12}$ defines a $\operatorname{Spin}(7, \mathbb{C})$-invariant inner product on $V$. So we have fundamental highest weight vectors $f_{0}, \varepsilon$ and corresponding (unnormalized) fundamental invariants $\gamma_{1}(z)=$ $|z|^{2}$ and $\gamma_{2}(z)=|\varepsilon(z)|^{2}$.

4.1.10. Spin(9) According to [11], there are three fundamental highest weight vectors, of degrees 1,2 and 2 . The space $\mathscr{P}_{2}(V)$ decomposes into three irreducible components, of dimensions 126,9 and 1 . The 9-dimensional irreducible is a copy of the standard representation of $\operatorname{SO}(9, \mathbb{C})$, and we have a $\operatorname{Spin}(9, \mathbb{C})$-invariant. More explicitly, we take $V=\Lambda\left(\mathbb{C}^{4}\right)$ and use the natural basis for $V$ with dual basis $f_{0}, f_{i}, f_{i j}, f_{i j k}, f_{1234}$ as in Example 4.1.9. Here $f_{0}$ is a highest weight vector for $\mathscr{P}_{1}(V) \cong V^{*}$, and $f_{0}^{2}$ is a highest weight vector for the 126-dimensional irreducible in $\mathscr{P}_{2}(V)$. A highest weight vector for the 9-dimensional irreducible is $f_{0} f_{234}$ $f_{2} f_{34}+f_{3} f_{24}-f_{4} f_{23}$, and the $\operatorname{Spin}(9, \mathbb{C})$-invariant inner product is given by the following pairing of coordinates:

$$
f_{0} f_{1234}+f_{1} f_{234}-f_{2} f_{134}+f_{3} f_{124}-f_{4} f_{123}-f_{12} f_{34}+f_{13} f_{24}-f_{14} f_{23} .
$$

4.1.11. $\operatorname{Spin}(10)$ The spin representation for $\operatorname{Spin}(10, \mathbb{C})$ can be realized in $V=\Lambda^{\text {even }}\left(\mathbb{C}^{5}\right)=\mathbb{C} \oplus \Lambda^{2}\left(\mathbb{C}^{5}\right) \oplus \Lambda^{4}\left(\mathbb{C}^{5}\right)$, and we use the natural bases for $V$ and $V^{*}$ as in the preceding two examples. We have fundamental highest weight vectors of degrees 1 and 2 given by $f_{0}$ and $f_{23} f_{45}-f_{24} f_{35}+f_{25} f_{34}$. The second of these generates a copy of the standard module for $\mathrm{SO}(10, \mathbb{C})$ in $\mathscr{P}_{2}(V)$.

4.1.12. $\mathbf{E}_{6}$ Following [7], we realize the standard representation of $E_{6}$ on a Jordan algebra $V$ of dimension 27 with elements

$$
X=\left[\begin{array}{lll}
\xi_{1} & x_{3} & \bar{x}_{2} \\
\bar{x}_{3} & \xi_{2} & x_{1} \\
x_{2} & \bar{x}_{1} & \xi_{3}
\end{array}\right],
$$


where $\xi_{i} \in \mathbb{C}$ and $x_{i} \in \mathscr{C}$, the 8-dimensional (complex) Cayley algebra. We have $\operatorname{so}(8, \mathbb{C})$ as a subalgebra of the the Lie algebra of $E_{6}$. Under the restriction to $s o(8, \mathbb{C})$, $V$ decomposes into three 8-dimensional irreducibles (the standard representation and the two inequivalent spin representations) and a 3-dimensional trivial subspace (corresponding to the diagonal entries in $X$ ).

Under an appropriate choice of positive roots, we find that $X \mapsto \xi_{1}$ is a highest weight vector for $\mathscr{P}_{1}(V)$. We are left with two remaining fundamental highest weight vectors, of degrees 2 and 3 . The highest weight vector in degree two generates the 27-dimensional representation contragredient to $\mathscr{P}_{1}(V)$. Let $V_{3}$ be the subspace of $V$ spanned by the matrices $X$ with $x_{3} \neq 0$, and all other entries zero. Then $\operatorname{so}(8, \mathbb{C})$ acts on $V_{3}$ by one of the spin representations. There is an $s o(8, \mathbb{C})$-invariant inner product on $V_{3}$ given by pairing the appropriate coordinates, and this is the fundamental highest weight vector of degree two. In degree three, we have an $E_{6}$-invariant, 'det', given explicitly by

$$
X \mapsto \xi_{1} \xi_{2} \xi_{3}+\left(x_{1} x_{2} x_{3}+\overline{x_{1} x_{2} x_{3}}\right)-\xi_{1} x_{1} \bar{x}_{1}-\xi_{2} x_{2} \bar{x}_{2}-\xi_{3} x_{3} \bar{x}_{3} .
$$

4.2. Indecomposable non-irreducible multiplicity free actions Table 2 lists the semisimple groups $G \subset \mathrm{GL}(V)$ acting indecomposably on $V=V_{1} \oplus V_{2}$ for which the action of $G \times\left(\mathbb{C}^{\times}\right)^{2}$ is multiplicity free. The subscripts on the direct sums indicate simple factors acting diagonally. Thus, for example, SL(n) $\oplus_{\mathbf{S L}(\mathbf{n})}(\mathbf{S L}(\mathbf{n}) \otimes \mathbf{S L}(\mathbf{m}))$ denotes the image of $\operatorname{SL}(n) \times \operatorname{SL}(m)$ under the representation on $V=V_{1} \oplus V_{2}=$ $\left(\mathbb{C}^{n}\right) \oplus\left(\mathbb{C}^{n} \otimes \mathbb{C}^{m}\right)$ where $\operatorname{SL}(n, \mathbb{C})$ acts diagonally on $V_{1}$ and $V_{2}$. In each case only one simple factor acts diagonally. For each such group $G$, the restrictions to $V_{1}$ and $V_{2}$ are irreducible multiplicity free actions and have thus been discussed above. In each case, we use the orthonormal basis for $V$ obtained by adjoining the standard orthonormal bases for $V_{1}$ and $V_{2}$, employed in our discussion of the irreducible multiplicity free actions. It is transparent that if we use such a basis to realize $G$ as a matrix group $G \subset \operatorname{GL}(n, \mathbb{C})$, then $\mathfrak{g}_{\mathbb{Q}}=\mathfrak{g} \cap g l(n, \mathbb{Q})$ is a rational form for $\mathfrak{g}$. In each case we present formulae for the fundamental highest weight vectors in $\mathbb{C}[V]$ that are rational in the coordinates with respect to this natural basis. Clearly, these include fundamental highest weight vectors for the actions of $G$ on $V_{1}$ and $V_{2}$. In each case, however, there are additional fundamental highest weights. We have listed the number of fundamental highest weight vectors and their degrees in Table 2. We follow the notational conventions in [5], to which we refer the reader for justification of the decompositions of $\mathbb{C}[V]$ described below.

4.2.1. SL(n) $\oplus_{\mathrm{SL}(\mathbf{n})} \mathrm{SL}(\mathbf{n}) \quad$ Here $G=\mathrm{SL}(n, \mathbb{C})$ acts on $V=V_{1} \oplus V_{2}=\mathbb{C}^{n} \oplus \mathbb{C}^{n}$ via two copies of its defining representation. The decomposition is

$$
\mathscr{P}_{k}\left(V_{1}\right) \otimes \mathscr{P}_{\ell}\left(V_{2}\right)=\sum_{j \leq \min (k, \ell)} \rho_{(k+\ell-j, j)}^{n} .
$$


TABle 2.

\begin{tabular}{|c|c|}
\hline $\begin{array}{l}\text { Group } \\
\text { Example number }\end{array}$ & $\begin{array}{l}\text { Degrees of fundamental highest } \\
\text { weight vectors (number) }\end{array}$ \\
\hline $\begin{array}{l}\mathbf{S L}(\mathbf{n}) \oplus_{\mathbf{S L}(\mathbf{n})} \mathbf{S L}(\mathbf{n})(n \geq 2) \\
\text { Example } 4.2 .1\end{array}$ & $1,1,2(3)$ \\
\hline $\begin{array}{l}\mathbf{S L}(\mathbf{n}) \oplus_{\mathbf{S L}(\mathbf{n})} \mathbf{S L}(\mathbf{n})^{*}(n \geq 3) \\
\text { Example } 4.2 .2\end{array}$ & $1,1,2(3)$ \\
\hline $\begin{array}{l}\mathbf{S L}(\mathbf{n}) \oplus_{\mathrm{SL}(\mathbf{n})} \Lambda^{2}(\mathbf{S L}(\mathbf{n}))(n \geq 4) \\
\text { Example } 4.2 .3\end{array}$ & $\begin{array}{l}1,2, \ldots,\lfloor n / 2\rfloor \\
1,2, \ldots,\lfloor(n+1) / 2\rfloor \\
(n)\end{array}$ \\
\hline $\begin{array}{l}\mathbf{S L}(\mathbf{n})^{*} \oplus_{\mathbf{S L}(\mathbf{n})} \Lambda^{2}(\mathbf{S L}(\mathbf{n}))(n \geq 4) \\
\text { Example } 4.2 .6\end{array}$ & $\begin{array}{l}1,2, \ldots,\lfloor n / 2\rfloor \\
1,2, \ldots,\lfloor(n-1) / 2\rfloor \\
(n-1)\end{array}$ \\
\hline $\begin{array}{l}\mathbf{S L}(\mathbf{n}) \oplus_{\mathbf{S L}(\mathbf{n})}(\mathbf{S L}(\mathbf{n}) \otimes \mathbf{S L}(\mathbf{m}))(n, m \geq 2) \\
\text { Example } 4.2 .4\end{array}$ & $\begin{array}{l}1,2, \ldots, \min (n, m) \\
1,2, \ldots, \min (n, m+1) \\
(\min (2 n, 2 m+1))\end{array}$ \\
\hline $\begin{array}{l}\mathbf{S L}(\mathbf{n})^{*} \oplus \mathbf{S L ( n )}(\mathbf{S L}(\mathbf{n}) \otimes \mathbf{S L}(\mathbf{m})) \\
(n \geq 3, m \geq 2) \\
\text { Example } 4.2 .5\end{array}$ & $\begin{array}{l}1,2, \ldots, \min (n, m) \\
1,2, \ldots, \min (n, m+1) \\
(\min (2 n, 2 m+1))\end{array}$ \\
\hline $\begin{array}{l}\mathbf{S L}(2) \oplus_{\mathbf{S L}(2)}(\mathbf{S L}(\mathbf{2}) \otimes \mathbf{S p}(\mathbf{2 n}))(n \geq 2) \\
\text { Example } 4.2 .7\end{array}$ & $1,1,2,2,2(5)$ \\
\hline $\begin{array}{l}(\mathbf{S L}(\mathbf{n}) \otimes \mathbf{S L}(2)) \oplus_{\mathbf{S L}(2)}(\mathbf{S L}(2) \otimes \mathbf{S L}(\mathbf{m})) \\
(n, m \geq 2) \\
\text { Example } 4.2 .8\end{array}$ & $1,1,2,2,2(5)$ \\
\hline $\begin{array}{l}(\mathbf{S L}(\mathbf{n}) \otimes \mathbf{S L}(2)) \oplus_{\mathbf{S L}(2)}(\mathbf{S L}(2) \otimes \mathbf{S p}(\mathbf{2 m})) \\
(n, m \geq 2) \\
\text { Example } 4.2 .8\end{array}$ & $1,1,2,2,2,2(6)$ \\
\hline $\begin{array}{l}(\mathbf{S p}(\mathbf{2 n}) \otimes \mathbf{S L}(2)) \oplus_{\mathrm{SL}(2)}(\mathbf{S L}(2) \otimes \mathbf{S p}(2 \mathrm{~m})) \\
(n, m \geq 2) \\
\text { Example } 4.2 .8\end{array}$ & $1,1,2,2,2,2,2(7)$ \\
\hline $\begin{array}{l}\mathbf{S p}(2 \mathbf{2 n}) \oplus_{\mathbf{S p}(2 \mathbf{n})} \mathbf{S p}(\mathbf{2 n})(n \geq 2) \\
\text { Example } 4.2 .9\end{array}$ & $1,1,2,2(4)$ \\
\hline $\begin{array}{l}\operatorname{Spin}(8) \oplus_{\operatorname{Spin}(8)} \mathbf{S O}(8) \\
\text { Example } 4.2 .10\end{array}$ & $1,1,2,2,2(5)$ \\
\hline
\end{tabular}


If we identify $V$ with $\mathbb{C}^{n} \otimes \mathbb{C}^{2}$, we can describe the fundamental highest weight vectors as $\zeta_{1}=z_{11}, \zeta_{1}^{\prime}=z_{12}$, and $\zeta_{2}=\operatorname{det}_{2}(z)$. The irreducible component $\rho_{(k+\ell-j, j)}^{n}$ has highest weight vector $\zeta_{1}^{k-j}\left(\zeta_{1}^{\prime}\right)^{\ell-j} \zeta_{2}^{j}$. The fundamental invariants are $\left|v_{1}\right|^{2},\left|v_{2}\right|^{2}$, and $\sum_{|I|=2}\left|\operatorname{det}_{l}(z)\right|^{2}$, up to normalizations.

4.2.2. $\mathbf{S L}(\mathbf{n}) \oplus_{\mathbf{S L}(\mathbf{n})} \mathrm{SL}(\mathbf{n})^{*} \quad$ Here $\operatorname{SL}(n, \mathbb{C})$ acts on $V=V_{1} \oplus V_{2}=\mathbb{C}^{n} \oplus\left(\mathbb{C}^{n}\right)^{*}$ and we have

$$
\mathscr{P}_{k}\left(V_{1}\right) \otimes \mathscr{P}_{\ell}\left(V_{2}\right)=\sum_{j \leq \min (k, \ell)} \rho_{(k+\ell-j, \ell, \ldots, \ell, j)}^{n} .
$$

Again, there are three fundamental highest weight vectors, of degrees 1,1 and 2 . The first two are coordinates in $V$ and $V^{*}$ respectively. The fundamental highest weight vector of degree two is given by the natural pairing $V_{1} \times V_{2}=\mathbb{C}^{n} \times\left(\mathbb{C}^{n}\right)^{*} \rightarrow \mathbb{C}$, which is $\operatorname{SL}(n)$-invariant. The irreducible component $\rho_{(k+\ell-j, \ell, \ldots, \ell, j)}^{n}$ has highest weight vector $\zeta_{1}^{k-j}\left(\zeta_{1}^{\prime}\right)^{\ell-j} \zeta_{2}^{j}$ as in the preceding example.

4.2.3. SL(n) $\oplus_{\mathbf{S L}(\mathbf{n})} \Lambda^{2}(\mathbf{S L}(\mathbf{n})) \quad$ We identify $V=V_{1} \oplus V_{2}=\mathbb{C}^{n} \oplus \Lambda^{2}\left(\mathbb{C}^{n}\right)$ with $\Lambda^{2}\left(\mathbb{C}^{n+1}\right)$ by regarding the first row (or column) of an $(n+1) \times(n+1)$ skew symmetric matrix as an element of $\mathbb{C}^{n}$, and the remaining entries as an element of $\Lambda^{2}\left(\mathbb{C}^{n}\right)$. For $z \in \Lambda^{2}\left(\mathbb{C}^{n+1}\right)$, we write $z^{\prime}$ for this element of $\Lambda^{2}\left(\mathbb{C}^{n}\right)$. That is, $z^{\prime}$ is obtained by removing the first row and column of $z$. Under this identification, the diagonal action of $\operatorname{SL}(n, \mathbb{C})$ on $V_{1} \oplus V_{2}$ is realized on $\Lambda^{2}\left(\mathbb{C}^{n+1}\right)$ by restricting the action of $\operatorname{SL}(n+1, \mathbb{C})$ to the subgroup $\operatorname{SL}(n, \mathbb{C}) \subset \operatorname{SL}(n+1, \mathbb{C})$ embedded as

$$
\left\{\left[\begin{array}{l|l}
1 & 0 \\
\hline 0 & A
\end{array}\right] \mid A \in \operatorname{SL}(n, \mathbb{C})\right\} .
$$

The fundamental highest weight vectors arising from the separate actions of $\operatorname{SL}(n, \mathbb{C})$ on $V_{1}$ and $V_{2}$ are $z_{12}$ and $\zeta_{k}^{\prime}(z)$ for $k=1, \ldots,\lfloor n / 2\rfloor$, where $\zeta_{k}^{\prime}(z)$ is the Pfaffian of the first $2 k$ rows and columns of the $n \times n$ matrix $z^{\prime}$. There are additional fundamental highest weight vectors, $\zeta_{k}(z)$ for $k=1, \ldots,\lfloor(n+1) / 2\rfloor$. These are the Pfaffians of the first $2 k$ rows and columns of the $(n+1) \times(n+1)$ matrix $z$. Note that $\zeta_{1}(z)=z_{12}$, so our fundamental highest weight vectors are the $\zeta_{k}$ 's together with the $\zeta_{k}^{\prime \prime}$ 's. The discussion in Example 4.1.5 shows how these Pfaffians can be written explicitly as rational polynomials in the matrix entries. The decomposition of $\mathbb{C}[V]$ is $\mathbb{C}[V]=\sum_{D} \rho_{D}$, where $D$ ranges over all Young's diagrams. For $D=\left(\lambda_{1}, \ldots, \lambda_{n}\right)$, a highest weight vector for $\rho_{D}$ in $\mathbb{C}[V]$ is

$$
\zeta_{1}^{\lambda_{1}-\lambda_{2}}\left(\zeta_{1}^{\prime}\right)^{\lambda_{2}-\lambda_{3}} \zeta_{2}^{\lambda_{3}-\lambda_{4}} \cdots \zeta_{m}^{\lambda_{n-1}-\lambda_{n}}\left(\zeta_{m}^{\prime}\right)^{\lambda_{n}}
$$

when $n=2 m$ is even, and

$$
\zeta_{1}^{\lambda_{1}-\lambda_{2}}\left(\zeta_{1}^{\prime}\right)^{\lambda_{2}-\lambda_{3}} \zeta_{2}^{\lambda_{3}-\lambda_{4}} \cdots \zeta_{m}^{\lambda_{n-2}-\lambda_{n-1}}\left(\zeta_{m}^{\prime}\right)^{\lambda_{n-1}-\lambda_{n}}\left(\zeta_{m+1}\right)^{\lambda_{n}}
$$

when $n=2 m+1$ is odd. 
4.2.4. SL(n) $\oplus_{\mathbf{S L}(\mathbf{n})}(\mathbf{S L}(\mathbf{n}) \otimes \mathbf{S L}(\mathbf{m})) \quad$ We realize $V=V_{1} \oplus V_{2}=\mathbb{C}^{n} \oplus\left(\mathbb{C}^{n} \otimes \mathbb{C}^{m}\right)$ as $\mathbb{C}^{n} \otimes \mathbb{C}^{m+1}$ with $\operatorname{SL}(n, \mathbb{C}) \times \operatorname{SL}(m, \mathbb{C})$ acting via its embedding in $\operatorname{SL}(n, \mathbb{C}) \times$ $\mathrm{SL}(m+1, \mathbb{C})$. For an $n \times(m+1)$ matrix $z$, write $z^{\prime}$ for the $n \times m$ matrix obtained by removing the first column. The fundamental highest weight vectors are $\zeta_{k}(z)=\operatorname{det}_{k}(z)$ for $k=1, \ldots, \min (n, m+1)$ and $\zeta_{k}^{\prime}(z)=\operatorname{det}_{k}\left(z^{\prime}\right)$ for $k=1, \ldots, \min (n, m)$. Here $\zeta_{1}(z)=z_{11}$ and the $\zeta_{k}^{\prime}(z)$ 's are the fundamental highest weight vectors that arise from the separate actions of $\operatorname{SL}(n, \mathbb{C}) \times \operatorname{SL}(m)$ on $V_{1}$ and $V_{2}$. The irreducible components of $\mathbb{C}[V]$ have the form $\rho_{\left(\lambda_{1}+\mu_{1}, \ldots, \lambda_{n}+\mu_{n}\right)}^{n} \otimes \rho_{\lambda}^{m}$ where $\lambda$ is a Young's diagram with at $\operatorname{most} \min (n, m)$ rows, and $\mu=\left(\mu_{1}, \ldots, \mu_{n}\right)$ satisfies $\mu_{j} \leq \lambda_{j-1}-\lambda_{j}$ for $j \geq 2$. The highest weight vector for this component is

$$
\zeta_{1}^{\mu_{1}} \zeta_{2}^{\mu_{2}} \cdots \zeta_{n}^{\mu_{n}}\left(\zeta_{1}^{\prime}\right)^{\lambda_{1}-\lambda_{2}-\mu_{2}}\left(\zeta_{2}^{\prime}\right)^{\lambda_{2}-\lambda_{3}-\mu_{3}} \cdots\left(\zeta_{n-1}^{\prime}\right)^{\lambda_{n-1}-\lambda_{n}-\mu_{n}}\left(\zeta_{n}^{\prime}\right)^{\lambda_{n}}
$$

when $n \leq m$ and

$$
\zeta_{1}^{\mu_{1}} \zeta_{2}^{\mu_{2}} \cdots \zeta_{m+1}^{\mu_{m+1}}\left(\zeta_{1}^{\prime}\right)^{\lambda_{1}-\lambda_{2}-\mu_{2}}\left(\zeta_{2}^{\prime}\right)^{\lambda_{2}-\lambda_{3}-\mu_{3}} \cdots\left(\zeta_{m-1}^{\prime}\right)^{\lambda_{m-1}-\lambda_{m}-\mu_{m}}\left(\zeta_{m}^{\prime}\right)^{\lambda_{m}-\mu_{m+1}}
$$

when $n>m$.

4.2.5. SL(n)* $\oplus_{\mathbf{S L}(\mathbf{n})}(\mathbf{S L}(\mathbf{n}) \otimes \mathbf{S L}(\mathbf{m})) \quad$ To decompose $\mathbb{C}[V]$ under the action of $\mathrm{SL}(n, \mathbb{C}) \times \operatorname{SL}(m, \mathbb{C})$ on $V=V_{1} \oplus V_{2}=\left(\mathbb{C}^{n}\right)^{*} \oplus\left(\mathbb{C}^{n} \otimes \mathbb{C}^{m}\right)$, we must decompose $\rho_{k^{n-1}}^{n} \otimes\left(\rho_{\lambda}^{n} \otimes \rho_{\lambda}^{m}\right)$, where $k^{n-1}$ denotes the Young's diagram with $k$ boxes in each of $n-1$ rows and $\lambda$ is a Young's diagram with at $\operatorname{most} \min (n, m)$ rows. We obtain $\sum_{\mu} \rho_{\mu_{1}+k, \ldots, \mu_{n-1}+k, \mu_{n}}^{n} \otimes \rho_{\lambda}^{m}$, where $\mu_{1}+\cdots+\mu_{n}=\lambda_{1}+\cdots+\lambda_{n}, \mu_{j} \leq \lambda_{j}$ for $j=1, \ldots, n-1,0 \leq \mu_{n}-\lambda_{n} \leq k$, and $\lambda_{1} \geq \mu_{1} \geq \lambda_{2} \geq \mu_{2} \geq \cdots \geq \mu_{n-1} \geq \lambda_{n}$. We write $z=\left(z_{i j}\right)$ for elements of $V_{2}=\mathbb{C}^{n} \otimes \mathbb{C}^{m}$ and $\xi$ for elements in $V_{1}=\left(\mathbb{C}^{n}\right)^{*}$. Let $z^{\prime}$ be the $(n+1) \times m$ matrix

$$
z^{\prime}=\left[\begin{array}{ccc}
\xi\left(v_{1}\right) & \cdots & \xi\left(v_{m}\right) \\
z
\end{array}\right],
$$

where $v_{i}$ is the $i$ 'th column of $z$. Then the action of $\operatorname{SL}(n, \mathbb{C}) \times \operatorname{SL}(m, \mathbb{C})$ on $z^{\prime}$ is given by embedding $\operatorname{SL}(n, \mathbb{C}) \times \operatorname{SL}(m, \mathbb{C})$ in $\operatorname{SL}(n+1, \mathbb{C}) \times \operatorname{SL}(m, \mathbb{C})$. The standard coordinate function $\xi_{n}$ is a highest weight vector for the representation of $\operatorname{SL}(n, \mathbb{C})$ on $\mathscr{P}_{1}\left(V_{1}\right) \cong V_{1}^{*} \cong \mathbb{C}^{n}$. Our fundamental highest weight vectors are thus $\xi_{n}, \operatorname{det}_{k}(z)$ $k=1, \ldots, \min (n, m)$ and $\operatorname{det}_{k}\left(z^{\prime}\right)$ for $k=1, \ldots, \min (n-1, m)$. Here $\operatorname{det}_{k}(z)$ is a polynomial of degree $k$ on $V$ and $\operatorname{det}_{k}\left(z^{\prime}\right)$ has degree $k+1$. A highest weight vector for the irreducible $\rho_{\mu_{1}+k, \ldots, \mu_{n-1}+k, \mu_{n}}^{n} \otimes \rho_{\lambda}^{m}$ in $\mathbb{C}[V]$ can be expressed in terms of the fundamental highest weight vectors as:

$$
\begin{aligned}
& \operatorname{det}_{1}\left(z^{\prime}\right)^{\lambda_{1}-\mu_{1}} \operatorname{det}_{2}\left(z^{\prime}\right)^{\lambda_{2}-\mu_{2}} \cdots \operatorname{det}_{n-1}\left(z^{\prime}\right)^{\lambda_{n-1}-\mu_{n-1}} \\
& \quad \times \operatorname{det}_{1}(z)^{\mu_{1}-\lambda_{2}} \operatorname{det}_{2}(z)^{\mu_{2}-\lambda_{3}} \cdots \operatorname{det}_{n}(z)^{\lambda_{n}} \xi_{n}^{k-\mu_{n}+\lambda_{n}} .
\end{aligned}
$$

We remark that the number and degrees of the fundamental highest weight vectors for this example coincide with those for the related Example 4.2.4. 
4.2.6. SL(n)* $\oplus_{\mathbf{S L}(\mathbf{n})} \Lambda^{2}(\mathbf{S L}(\mathbf{n})) \quad$ Here $V=V_{1} \oplus V_{2}=\left(\mathbb{C}^{n}\right)^{*} \oplus \Lambda^{2}\left(\mathbb{C}^{n}\right)$. We write $z=\left(z_{i j}\right) \in \Lambda^{2}\left(\mathbb{C}^{n}\right), \xi=\left(\xi_{i}\right) \in\left(\mathbb{C}^{n}\right)^{*}$, and let

$$
z^{\prime}=\left[\begin{array}{c|ccc}
0 & \xi\left(v_{1}\right) & \cdots & \xi\left(v_{n}\right) \\
\hline-\xi\left(v_{1}\right) & & & \\
\vdots & & z & \\
-\xi\left(v_{n}\right) & & &
\end{array}\right] \in \Lambda^{2}\left(\mathbb{C}^{n+1}\right),
$$

where $v_{i}$ is the $i$ 'th column of $z$. The group $\operatorname{SL}(n, \mathbb{C})$ acts on $z^{\prime}$ via the embedding $\operatorname{SL}(n, \mathbb{C}) \hookrightarrow \operatorname{SL}(n+1, \mathbb{C})$, as in Example 4.2.3. Our fundamental highest weight vectors are $\xi_{n}, \zeta_{k}$ for $k=1, \ldots,\lfloor(n-1) / 2\rfloor$ and $\zeta_{k}^{\prime}$ for $k=1, \ldots,\lfloor n / 2\rfloor$. Here $\zeta_{k}$ and $\zeta_{k}^{\prime}$ are the Pfaffians of the first $2 k$ rows and columns of $z$ and $z^{\prime}$ respectively. Note that $\zeta_{k}$ is a homogeneous polynomial of degree $k$ on $V$, whereas $\zeta_{k}^{\prime}$ has degree $k+1$.

To understand the decomposition of $\mathbb{C}[V]$, we consider the cases $n$ even and $n$ odd separately. If $n=2 m$, we need to consider the decomposition of $\rho_{k^{n-1}}^{n} \otimes \rho_{\lambda}^{n}$ where $\lambda=\left(\lambda_{1}, \lambda_{1}, \lambda_{2}, \lambda_{2}, \ldots, \lambda_{m}, \lambda_{m}\right)$. We obtain irreducible components corresponding to Young's diagrams $\left(k+\lambda_{1}, k+\mu_{1}, \ldots, k+\lambda_{m}, \mu_{m}+\lambda_{m}\right)$, where $\lambda_{1} \geq \mu_{1} \geq \lambda_{2} \geq$ $\mu_{2} \geq \cdots \geq \mu_{m-1} \geq \lambda_{m}, \mu_{m} \leq k$ and $\mu_{1}+\cdots+\mu_{m}=\lambda_{1}+\cdots \lambda_{m-1}$. The highest weight vector for this component is

$$
\left(\zeta_{1}^{\prime}\right)^{\lambda_{1}-\mu_{1}}\left(\zeta_{2}^{\prime}\right)^{\lambda_{2}-\mu_{2}} \cdots\left(\zeta_{m-1}^{\prime}\right)^{\lambda_{m-1}-\mu_{m-1}} \xi_{n}^{k-\mu_{m}} \zeta_{1}^{\mu_{1}-\lambda_{2}} \zeta_{2}^{\mu_{2}-\lambda_{3}} \cdots \zeta_{m-1}^{\mu_{m-1}-\lambda_{m}} \zeta_{m}^{\lambda_{m}}
$$

For $n=2 m+1$, we have $\rho_{k^{2 m}}^{n} \otimes \rho_{\lambda}^{n}$ with $\lambda=\left(\lambda_{1}, \lambda_{1}, \lambda_{2}, \lambda_{2}, \ldots, \lambda_{m}, \lambda_{m}, 0\right)$, whose irreducible components correspond to diagrams $\left(k+\lambda_{1}, k+\mu_{1}, \ldots, k+\lambda_{m}, k+\right.$ $\left.\mu_{m}, \mu_{m+1}\right)$, where $\lambda_{1} \geq \mu_{1} \geq \lambda_{2} \geq \mu_{2} \geq \cdots \geq \lambda_{m} \geq \mu_{m}, \mu_{m+1} \leq k$, and $\mu_{1}+\cdots+$ $\mu_{m+1}=\lambda_{1}+\cdots \lambda_{m}$. This component has highest weight vector

$$
\left(\zeta_{1}^{\prime}\right)^{\lambda_{1}-\mu_{1}}\left(\zeta_{2}^{\prime}\right)^{\lambda_{2}-\mu_{2}} \cdots\left(\zeta_{m}^{\prime}\right)^{\lambda_{m}-\mu_{m}} \xi_{n}^{k-\mu_{m+1}} \zeta_{1}^{\mu_{1}-\lambda_{2}} \zeta_{2}^{\mu_{2}-\lambda_{3}} \cdots \zeta_{m-1}^{\mu_{m-1}-\lambda_{m}} \zeta_{m}^{\mu_{m}}
$$

It is interesting to note that here we have one fewer fundamental highest weight vector than in the related untwisted Example 4.2.3.

4.2.7. SL(2) $\oplus_{\mathbf{S L}(2)}(\mathbf{S L}(2) \otimes \mathbf{S p}(2 \mathrm{n})) \quad$ We identify $V=V_{1} \oplus V_{2}=\mathbb{C}^{2} \oplus\left(\mathbb{C}^{2 n} \otimes \mathbb{C}^{2}\right)$ with $\mathbb{C}^{2 n+1} \otimes \mathbb{C}^{2}$ as in Example 4.2.4, and embed $G=\operatorname{Sp}(2 n, \mathbb{C}) \times \operatorname{SL}(2, \mathbb{C})$ in $\operatorname{SL}(2 n+1, \mathbb{C}) \times \operatorname{SL}(2, \mathbb{C})$. Write $z=\left(z_{i j}\right)$ for a $(2 n+1) \times 2$ matrix in $V$ and let $z^{\prime}$ denote the $2 n \times 2$ matrix obtained by removing the first row of $z$. The fundamental highest weight vectors are $z_{11}, z_{21}, \varepsilon^{\prime}, \operatorname{det}_{2}(z)$ and $\operatorname{det}_{2}\left(z^{\prime}\right)$, where $\varepsilon^{\prime}$ is the symplectic product of the columns of $z^{\prime}$. The irreducible components are of the form

$$
\rho_{\left(\alpha_{1}+\alpha_{3}+\beta_{1}, \alpha_{2}+\alpha_{3}+\beta_{2}\right)}^{n} \otimes \sigma_{\alpha_{1}, \alpha_{2}} \subset \mathscr{P}_{\beta_{1}+\beta_{2}}\left(\mathbb{C}^{2}\right) \otimes \mathscr{P}_{\alpha_{1}+\alpha_{2}+2 \alpha_{3}}\left(\mathbb{C}^{2 n} \otimes \mathbb{C}^{2}\right)
$$

with the restrictions $\alpha_{1} \geq \alpha_{2}, \beta_{2} \leq \alpha_{1}-\alpha_{2}$. The highest weight vector for this component is

$$
z_{11}^{\beta_{1}} z_{12}^{\alpha_{1}-\alpha_{2}-\beta_{2}}\left(\varepsilon^{\prime}\right)^{\alpha_{3}} \operatorname{det}_{2}(z)^{\beta_{2}} \operatorname{det}_{2}\left(z^{\prime}\right)^{\alpha_{2}}
$$


4.2.8. $(\mathbf{S L}(\mathbf{n}) \otimes \mathbf{S L}(2)) \oplus_{\mathbf{S L}(2)}(\mathbf{S L}(2) \otimes \mathbf{S L}(\mathrm{m})) \quad$ Here we examine together the actions of

(a) $G=\operatorname{SL}(n, \mathbb{C}) \times \operatorname{SL}(2, \mathbb{C}) \times \operatorname{SL}(m, \mathbb{C})$ on $V=V_{1} \oplus V_{2}=\left(\mathbb{C}^{n} \otimes \mathbb{C}^{2}\right) \oplus\left(\mathbb{C}^{2} \otimes \mathbb{C}^{m}\right)$,

(b) $G=\operatorname{SL}(n, \mathbb{C}) \times \operatorname{SL}(2, \mathbb{C}) \times \operatorname{Sp}(2 m, \mathbb{C})$ on $V=V_{1} \oplus V_{2}=\left(\mathbb{C}^{n} \otimes \mathbb{C}^{2}\right) \oplus\left(\mathbb{C}^{2} \otimes \mathbb{C}^{2 m}\right)$,

(c) $G=\operatorname{Sp}(2 n, \mathbb{C}) \times \operatorname{SL}(2, \mathbb{C}) \times \operatorname{Sp}(2 m, \mathbb{C})$ on $V=V_{1} \oplus V_{2}=\left(\mathbb{C}^{2 n} \otimes \mathbb{C}^{2}\right) \oplus\left(\mathbb{C}^{2} \otimes \mathbb{C}^{2 m}\right)$.

In each case, we identify $V$ with $\mathbb{C}^{N+M} \otimes \mathbb{C}^{2}$, where $N=n$ or $2 n, M=m$ or $2 m$. We write elements of $V$ as

$$
z=\left[\begin{array}{l}
x \\
y
\end{array}\right]
$$

where $x \in \mathbb{C}^{N} \otimes \mathbb{C}^{2}$ and $y \in \mathbb{C}^{M} \otimes \mathbb{C}^{2}$. In cases (b) and (c), we have the symplectic invariants $\varepsilon(x), \varepsilon(y)$ given by the symplectic inner products of the columns of $x$ and $y$ respectively.

In case (a), $\mathbb{C}[V]$ decomposes into irreducibles of the form $\rho_{\lambda}^{n} \otimes \rho_{v}^{2} \otimes \rho_{v}^{m}$ where $|\mu|=|\lambda|+|\nu|, \mu_{2} \leq \lambda_{1}+\nu_{2}, \mu_{1}-\lambda_{1} \geq v_{2}$, and $\mu_{2} \geq \lambda_{2}+\nu_{2}$. The fundamental highest weight vectors are $x_{11}, y_{11}, \operatorname{det}_{2}(x), \operatorname{det}_{2}(y)$, and $x_{11} y_{12}-x_{12} y_{11}$. The irreducible component above has highest weight vector

$$
x_{11}^{\lambda_{1}+\nu_{2}-\mu_{2}} y_{11}^{\mu_{1}-\lambda_{1}-\nu_{2}} \operatorname{det}_{2}(x)^{\lambda_{2}} \operatorname{det}_{2}(y)^{\nu_{2}}\left(x_{11} y_{12}-x_{12} y_{11}\right)^{\mu_{2}-\nu_{2}-\lambda_{2}} \text {. }
$$

In case (b), the irreducibles are of the form

$$
\rho_{\lambda}^{n} \otimes \rho_{\mu}^{2} \otimes \sigma_{v}^{m} \subset \mathscr{P}_{|\lambda|}\left(\mathbb{C}^{n} \otimes \mathbb{C}^{2}\right) \otimes \mathscr{P}_{|\nu|+2 j}\left(\mathbb{C}^{2 m} \otimes \mathbb{C}^{2}\right),
$$

where $j \geq 0,|\mu|=|\lambda|+|v|+2 j, \mu_{1} \geq v_{1}+j, \lambda_{2}+v_{2}+j \leq \mu_{2} \leq v_{1}+\lambda_{2}+j$. The fundamental highest weight vectors are $x_{11}, y_{11}, \operatorname{det}_{2}(x), \operatorname{det}_{2}(y), \varepsilon(y)$ and $x_{11} y_{12}-$ $x_{12} y_{11}$. The typical irreducible component above has highest weight vector

$$
x_{11}^{\lambda_{1}+\nu_{2}+j-\mu_{2}} y_{11}^{\nu_{1}+\lambda_{2}+j-\mu_{2}} \operatorname{det}_{2}(x)^{\lambda_{2}} \operatorname{det}_{2}(y)^{\nu_{2}} \varepsilon(y)^{j}\left(x_{11} y_{12}-x_{12} y_{11}\right)^{\mu_{2}-\nu_{2}-\lambda_{2}-j} .
$$

In case (c), the fundamental highest weight vectors are $x_{11}, y_{11}, \operatorname{det}_{2}(x), \operatorname{det}_{2}(y)$, $\varepsilon(x), \varepsilon(y)$ and $x_{11} y_{12}-x_{12} y_{11}$. The irreducibles are of the form

$$
\sigma_{\lambda}^{n} \otimes \rho_{\mu}^{2} \otimes \sigma_{v}^{m} \subset \mathscr{P}_{|\lambda|+2 a}\left(\mathbb{C}^{2 n} \otimes \mathbb{C}^{2}\right) \otimes \mathscr{P}_{|\nu|+2 b}\left(\mathbb{C}^{2 m} \otimes \mathbb{C}^{2}\right),
$$

where $|\mu|=|\lambda|+|\nu|+2 a+2 b, \mu_{2} \geq \lambda_{2}+\nu_{2}+a+b, \mu_{2} \leq \lambda_{1}+\nu_{2}+a+b$ and $\mu_{2} \leq \lambda_{2}+v_{1}+a+b$. This has highest weight vector

$$
x_{11}^{\lambda_{1}+v_{2}+a+b-\mu_{2}} y_{11}^{\nu_{1}+\lambda_{2}+a+b-\mu_{2}} \operatorname{det}_{2}(x)^{\lambda_{2}} \operatorname{det}_{2}(y)^{\nu_{2}} \varepsilon(x)^{a} \varepsilon(y)^{b}\left(x_{11} y_{12}-x_{12} y_{11}\right)^{\mu_{2}-\nu_{2}-\lambda_{2}-a-b} .
$$


4.2.9. Sp(2n) $\oplus_{\mathbf{S p}(2 \mathrm{n})} \mathbf{S p}(\mathbf{2 n}) \quad$ We identify $V=V_{1} \oplus V_{2}=\mathbb{C}^{2 n} \oplus \mathbb{C}^{2 n}$ with $\mathbb{C}^{2 n} \otimes \mathbb{C}^{2}$ and write points $z \in V$ as $z=\left(z_{i j}\right)$. The group $G=\operatorname{Sp}(2 n, \mathbb{C})$ acts on $V$ from the left and the symplectic product $\varepsilon(z)$ of the columns is an invariant. The fundamental highest weight vectors are $z_{11}, z_{12}, \varepsilon(z)$, and $\operatorname{det}_{2}(z)$. The irreducible components of $\mathscr{P}_{k}\left(\mathbb{C}^{2 n}\right) \otimes \mathscr{P}_{\ell}\left(\mathbb{C}^{2 n}\right)$ are of the form $\sigma_{\lambda_{1}, \lambda_{2}}^{n}$, with $\lambda_{1} \geq \lambda_{2}$, and $\lambda_{1}+\lambda_{2}=k+\ell-2 m$ for $m \geq 0$. The highest weight vector for this irreducible is

$$
z_{11}^{k-\lambda_{2}-m} z_{12}^{\ell-\lambda_{2}-m} \varepsilon(z)^{m} \operatorname{det}_{2}(z)^{\lambda_{2}} .
$$

4.2.10. $\operatorname{SO}(8) \oplus_{\operatorname{Spin}(8)} \operatorname{Spin}(8)$ In this example, $\operatorname{Spin}(8, \mathbb{C})$ acts on $V=$ $V_{1} \oplus V_{2}=\mathbb{C}^{8} \oplus \Lambda^{\text {even }}\left(\mathbb{C}^{4}\right)$ via the standard representation of $\operatorname{SO}(8, \mathbb{C})$ on $V_{1}$ and the spin representation on $V_{2} \cong \mathbb{C}^{8}$. These two representations can be written as $\omega_{1000}$ and $\omega_{\frac{1}{2} \frac{1}{2} \frac{1}{2} \frac{1}{2}}$, where the subscripts indicate the highest weights for the representations. We have the decompositions

$$
\begin{aligned}
\mathscr{P}_{\ell}\left(V_{1}\right) \otimes \mathscr{P}_{2 k}\left(V_{2}\right) & =\sum_{i, j, m} \omega_{k+\ell-i-2 j-m, k-i, k-i, k-i-m}, \quad \text { and } \\
\mathscr{P}_{\ell}\left(V_{1}\right) \otimes \mathscr{P}_{2 k+1}\left(V_{2}\right) & =\sum_{i, j, m} \omega_{k+\ell-i-2 j-m+\frac{1}{2}, k-i+\frac{1}{2}, k-i+\frac{1}{2}, k-i-m+\frac{1}{2}} .
\end{aligned}
$$

We use the standard basis for $V_{2}^{*}$ as in Example 4.1.11 and give $V_{1}$ coordinates $z_{1}, \ldots, z_{8}$, which are weight vectors for the maximal torus. We have fundamental highest weight vectors corresponding to the highest weight vectors for each $V_{i}$, the $\operatorname{Spin}(8, \mathbb{C})$-invariants in each $V_{i}$ and a fifth highest weight vector which generates the other spin representation in $\mathscr{P}_{2}(V)$. Explicitly, these are

$$
\begin{array}{ll}
\zeta_{1}=z_{1}, \quad \zeta_{1}^{\prime}=f_{0}, & \zeta_{2}=z_{1} z_{5}+z_{2} z_{6}+z_{3} z_{7}+z_{4} z_{8}, \\
\zeta_{2}^{\prime}=f_{0} f_{1234}-f_{12} f_{34}+f_{13} f_{24}-f_{14} f_{13}, & \zeta_{2}^{\prime \prime}=z_{8} f_{0}+z_{1} f_{14}+z_{2} f_{24}+z_{3} f_{34} .
\end{array}
$$

The irreducible component $\omega_{k+\ell-i-2 j-m, k-i, k-i, k-i-m}$ has highest weight vector

$$
\zeta_{1}^{\ell-2 j-m} \zeta_{2}^{j}\left(\zeta_{1}^{\prime}\right)^{2 k-2 i-m}\left(\zeta_{2}^{\prime}\right)^{i}\left(\zeta_{2}^{\prime \prime}\right)^{m}
$$

and $\omega_{k+\ell-i-2 j-m+\frac{1}{2}, k-i+\frac{1}{2}, k-i+\frac{1}{2}, k-i-m+\frac{1}{2}}$ has highest weight vector

$$
\zeta_{1}^{\ell-2 j-m} \zeta_{2}^{j}\left(\zeta_{1}^{\prime}\right)^{2 k-2 i-m+1}\left(\zeta_{2}^{\prime}\right)^{i}\left(\zeta_{2}^{\prime \prime}\right)^{m}
$$

\section{References}

[1] C. Benson, J. Jenkins, R. Lipsman and G. Ratcliff, 'A geometric criterion for Gelfand pairs associated with the Heisenberg group', Pacific J. Math. 178 (1997), 1-36. 
[2] C. Benson, J. Jenkins and G. Ratcliff, 'Bounded $K$-spherical functions on Heisenberg groups', $J$. Funct. Anal. 105 (1992), 409-443.

[3] _ , ' $O(n)$-spherical functions on Heisenberg groups', Contemp. Math. 145 (1993), 181-197.

[4] - 'The spherical transform of a Schwartz function on the Heisenberg group', J. Funct. Anal. 154 (1998), 379-423.

[5] C. Benson and G. Ratcliff, 'A classification for multiplicity free actions', J. Algebra 181 (1996), $152-186$.

[6] - 'Combinatorics and spherical functions on the Heisenberg group', Representation Theory 2 (1998), 79-105.

[7] C. Chevalley and R. Shafer, 'The exceptional Lie algebras $F_{4}$ and $E_{6}$ ', Proc. Nat. Acad. Sci. Amer. 36 (1950), 137-141.

[8] H. Dib, 'Fonctions de Bessel sur une algèbre de Jordan', J. Math. Pures Appl. 69 (1990), 403-448.

[9] J. Faraut and A. Koranyi, Analysis on symmetric cones (Oxford University Press, New York, 1994).

[10] G. Folland, Harmonic analysis in phase space (Princeton University Press, New Jersey, 1989).

[11] R. Howe and T. Umeda, 'The Capelli identity, the double commutant theorem and multiplicity-free actions', Math. Ann. 290 (1991), 565-619.

[12] V. Kac, 'Some remarks on nilpotent orbits', J. Algebra 64 (1980), 190-213.

[13] A. Leahy, 'A classification of multiplicity free representations', J. Lie Theory 8 (1998), 367-391.

[14] A. Okounkov and G. Olshanski, 'Shifted Jack polynomials, binomial formula, and applications', Math. Res. Letters 4 (1997), 69-78.

[15] _ - 'Shifted Schur functions II. The binomial formula for characters of classical groups and its applications', Amer. Math. Soc. Transl. Ser. 2181 (1998), 245-271.

[16] S. Sahi, 'The spectrum of certain invariant differential operators associated to Hermitian symmetric spaces', in: Lie theory and geometry (ed. J. L. Brylinski), Progress in Math. 123 (Birkhäuser, Boston, 1994) pp. 569-576.

[17] Z. Yan, 'Special functions associated with multiplicity-free representations', preprint.

[18] _ 'Generalized hypergeometric functions and Laguerre polynomials in two variables', Contemp. Math. 138 (1992), 239-259.

Department of Mathematics and Computer Science

University of Missouri-St. Louis

St. Louis, MO 63121

USA

e-mail: benson@arch.umsl.edu, ratcliff@arch.umsl.edu 